\title{
Structure-guided glyco-engineering of ACE2 for improved potency as soluble SARS-CoV-2 decoy receptor
}

Tümay Capraz, ${ }^{1} \dagger$ Nikolaus F. Kienzl, ${ }^{2} \dagger$ Elisabeth Laurent, ${ }^{3} \dagger$ Jan W. Perthold, ${ }^{1}$ Esther FöderlNiederhöfer, ${ }^{7}$ Gerald Wirnsberger, ${ }^{7}$ Ali Mirazimi, ${ }^{6,8}$ Kurt Zatloukal, ${ }^{4}$ Lukas Mach, ${ }^{2}$ Josef M. Penninger, ${ }^{9,10} *$ Chris Oostenbrink, ${ }^{1} *$ Johannes Stadlmann ${ }^{5,9} *$

${ }^{1}$ Institute for Molecular Modeling and Simulation, University of Natural Resources and Life Sciences (BOKU), Muthgasse 18, 1190 Vienna, Austria.

${ }^{2}$ Institute of Plant Biotechnology and Cell Biology, Department of Applied Genetics and Cell Biology, University of Natural Resources and Life Sciences (BOKU), Muthgasse 18, 1190 Vienna, Austria.

205 Institute of Biochemistry, Department of Chemistry, University of Natural Resources and Life

3 Institute of Molecular Biotechnology, Department of Biotechnology and Core Facility Biomolecular \& Cellular Analysis, University of Natural Resources and Life Sciences (BOKU), Muthgasse 18, 1190 Vienna, Austria.

4 Diagnostic and Research Institute of Pathology, Medical University of Graz, Neue Stiftingtalstrasse 6, 8010 Graz, Austria. Sciences (BOKU), Muthgasse 18, 1190 Vienna, Austria.

${ }^{6}$ Karolinska Institute, Department of Laboratory Medicine, 17177 Stockholm, Sweden.

${ }^{7}$ Apeiron Biologics, Campus Vienna Biocenter 5, 1030 Vienna, Austria.

${ }^{8}$ National Veterinary Institute, 75189 Uppsala, Sweden.

$25{ }^{9}$ IMBA - Institute of Molecular Biotechnology of the Austrian Academy of Sciences, Dr. Bohr Gasse 3, 1030 Vienna, Austria.

${ }^{10}$ Department of Medical Genetics, Life Sciences Institute, University of British Columbia, Vancouver Campus, 2350 Health Sciences Mall, Vancouver, BC V6T 1Z3, Canada.

$30 \quad$ †equal contributions.

*Correspondence to:

j.stadlmann@boku.ac.at, chris.oostenbrink@boku.ac.at, lukas.mach@boku.ac.at, josef.penninger@ubc.ca

Running title: Glycoengineering of ACE2 improves SARS-CoV-2 neutralization. 


\section{Abstract}

Infection and viral entry of SARS-CoV-2 crucially depends on the binding of its Spike protein to angiotensin converting enzyme 2 (ACE2) presented on host cells. Glycosylation of both proteins is critical for this interaction. Recombinant soluble human ACE2 can neutralize SARS-CoV-2 and is currently undergoing clinical tests for the treatment of COVID-19. We used 3D structural models and molecular dynamics simulations to define the ACE2 N-glycans that critically influence Spike-ACE2 complex formation. Engineering of ACE2 N-glycosylation by sitedirected mutagenesis or glycosidase treatment resulted in enhanced binding affinities and improved virus neutralization without notable deleterious effects on the structural stability and catalytic activity of the protein. Importantly, simultaneous removal of all accessible N-glycans from recombinant soluble human ACE2 yields a superior SARS-CoV-2 decoy receptor with promise as effective treatment for COVID-19 patients. 


\section{Introduction}

The rapid spread of Severe Acute Respiratory Syndrome Coronavirus-2 (SARS-CoV-2), the causative pathogen of human coronavirus disease 2019 (COVID-19), has resulted in an unprecedented pandemic and worldwide health crisis. Similar to the beta-coronaviruses SARS$\mathrm{CoV}$ and Middle Eastern Respiratory Syndrome (MERS)-CoV, SARS-CoV-2 is highly transmissible and can lead to lethal pneumonia and multi-organ failure. ${ }^{1}$ For infection and viral

60 entry, the Spike surface protein of SARS-CoV-2 binds to angiotensin converting enzyme 2 (ACE2) on host cells. ${ }^{2,3}$ Recombinant soluble human ACE2 (rshACE2) has been shown to bind Spike, ${ }^{4}$ can effectively neutralize SARS-CoV-2 infections, ${ }^{5,6}$ and the corresponding drug candidate APN01 has undergone a phase 2 clinical trial for the treatment of hospitalized cases of COVID-19 (ClinicalTrials.gov Identifier: NCT04335136). A first case study of its use in a patient has been reported recently. ${ }^{7}$ Additionally, an aerosol formulation of APN01 has been developed and is currently undergoing Phase I clinical studies.

Multiple other therapeutic strategies attempt to target the Spike-ACE2 interaction, e.g. by development of neutralizing antibodies blocking the ACE2-binding site $^{8}$ or lectins that bind to glycans on the Spike surface. ${ }^{9,10}$ Using soluble ACE2 as a decoy receptor for Spike is particularly attractive, as it minimizes the risk that variants of concern may evade the treatment through mutations as has been observed for antibodies. ${ }^{11-13}$ Furthermore, protein engineering has yielded ACE2 variants with substantially improved affinities for Spike. ${ }^{14,15}$ Hence, soluble ACE2 based therapeutics offer considerable advantages over other therapeutic formats that aim to hamper the Spike-ACE2 interaction sterically.

75 Modern structural biology has been amazingly fast to respond to this pandemic. A mere three months after identification of SARS-CoV-2 as the etiologic agent of COVID-19, structures of the complex between ACE2 and the receptor binding domain (RBD) ${ }^{4,16,17}$ and of the ectodomain of trimeric Spike ${ }^{18,19}$ were already solved by X-ray crystallography or cryo-electron microscopy. While this provided unprecedented insight into the protein-protein interactions between Spike and ACE2, the structural impact of protein-bound glycans on the Spike-ACE2 interface could not be assessed experimentally so far due to their compositional diversity and conformational flexibility. Here, in silico modeling of the glycans offers a powerful alternative to study the 
effects of individual Spike and ACE2 glycans on the molecular interactions between these two proteins.

The SARS-CoV-2 Spike protein is heavily glycosylated with both complex and oligomannosidic type $\mathrm{N}$-glycans, ${ }^{1,10,20,21}$ thereby shielding a large portion of the protein surface. ${ }^{22-24}$ Similarly, ACE2 is a glycoprotein with up to seven highly utilized sites of N-glycosylation. ${ }^{21}$ Recent computational studies started to investigate protein glycosylation in the context of the interaction between Spike and ACE2..$^{21,22,25}$ Extensive all-atom molecular dynamics (MD) simulations indicated that Spike N-glycans attached to N165 and N234 could be important stabilizers of the ligand-accessible conformation of the receptor binding domain (RBD). ${ }^{22}$ Furthermore, it has been proposed that the N-glycan at position N343 acts as a gate facilitating RBD opening. ${ }^{26}$ Other MD studies concluded that the glycans attached to N90 and N322 of ACE2 could be major determinants of Spike binding, ${ }^{25}$ while yet other simulation works postulate that glycosylation does not affect the RBD-ACE2 interaction significantly. ${ }^{27,28}$ Genetic or pharmacological blockade of N-glycan biosynthesis at the oligomannose stage in ACE2expressing target cells was found to dramatically reduce viral entry, ${ }^{29}$ even though several glycoforms of ACE2 were found to display comparatively moderate variation with respect to Spike binding. ${ }^{30}$ Hence, a detailed understanding on how individual glycans on both Spike and 100 ACE2 influence their interaction and a comprehensive experimental validation of the MD findings is crucial for the rational design of novel therapeutic soluble ACE2 variants with enhanced Spike binding affinity and the capacity to block viral entry more efficiently than the native enzyme. ${ }^{21}$ The identification of the Spike glycans essential for efficient association with ACE2 will be also critical to guide rational design of improved SARS-CoV-2 vaccines.

\section{Results}

We started our research by creating 3D models of the trimeric Spike in complex with human ACE2 (hACE2). The RBD of Spike exists in two distinct conformations, referred to as "up" and "down". ${ }^{18,19}$ The "up" conformation corresponds to the receptor-accessible state with the RBD of one monomer exposed. By superimposing the RBD from the RBD-hACE2 complex ${ }^{17}$ with the 110 single RBD in the "up" conformation (monomer 3) of the trimeric Spike, ${ }^{18}$ an initial model was obtained. To assess the impact of all seven individual N-glycosylation sites of hACE2 on its interaction with Spike, we first elucidated the entire glycome of rshACE2 (Fig. S1). This also 
provided information on the glycans attached to N690, a glycosylation site not covered in previous glycoproteomic studies of soluble hACE2. ${ }^{21,30}$ For recombinant trimeric Spike the glyco-analysis has been reported elsewhere. ${ }^{10,20,21,31}$ Based on the site-specific glycosylation profiles we added complex or oligo-mannosidic glycan trees to the respective sites of Spike and ACE2 (Table S1). We hence constructed fully glycosylated atomistic models of the trimeric Spike glycoprotein, free dimeric ACE2 and of the Spike glycoprotein in complex with dimeric hACE2 (Fig. 1). Using these fully glycosylated structures, we performed molecular dynamics simulations of the Spike-ACE2 complex (Video S1), and of free hACE2. Inspection of the most important interacting residues on Spike and ACE2, their average distances and the electrostatic potential of the interface area identified critical contact sites (Figs. S2 and S3).
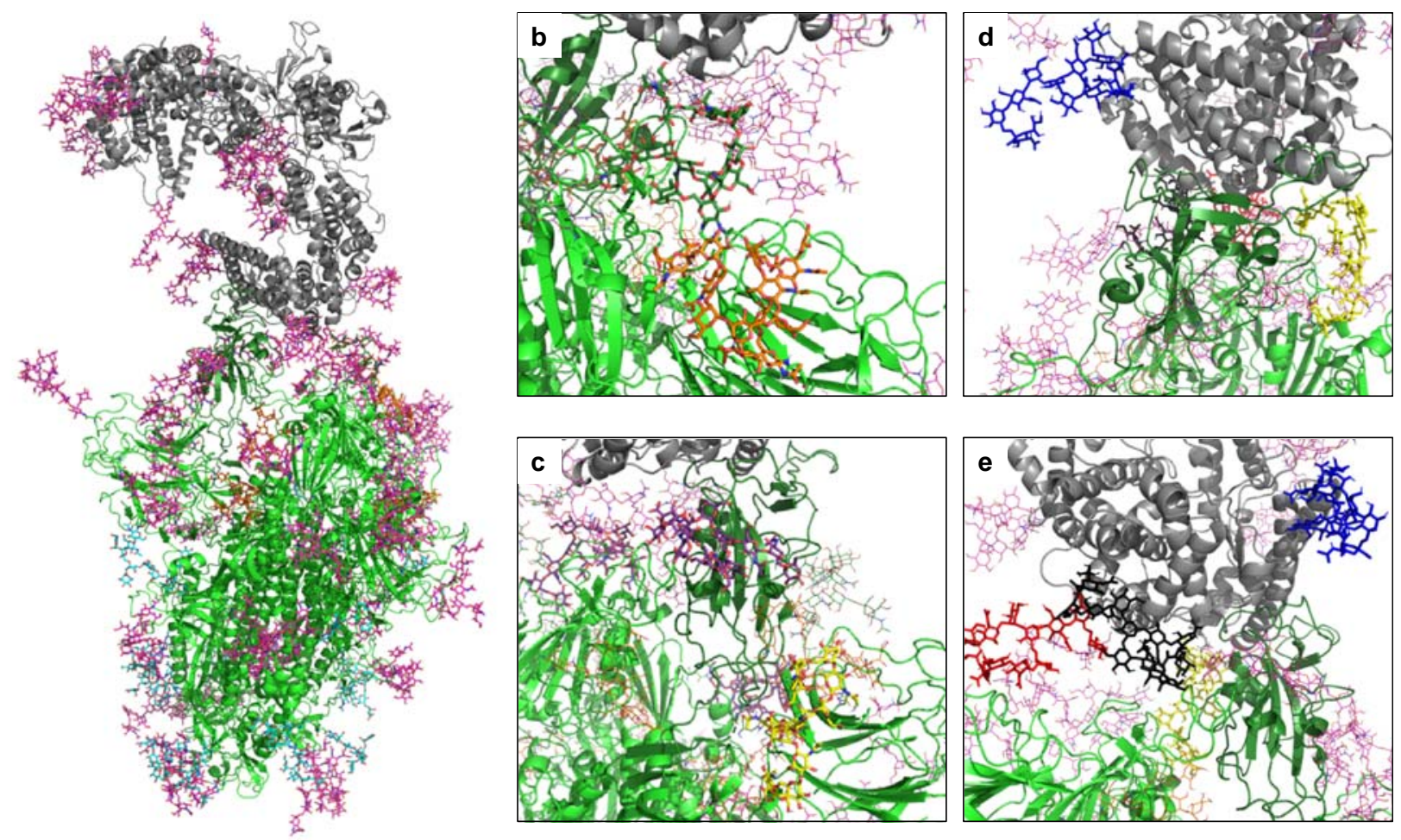

Figure 1. A 3D structural model of the glycosylated Spike-hACE2 complex. (a) 3D model of the Spike trimer (in green, with RBD of monomer 3 in dark green) binding to ACE2 (in grey) with complex glycosylation in magenta, Man5 glycans in light blue and Man9 glycans in orange. (b) Close-up view of the glycans at N122 (orange sticks) and N165 (dark green sticks) on monomer 3 of Spike. (c) Close-up view of the glycans at N331 (yellow sticks) and N343 (purple sticks) on monomer 3 of Spike. (d) Close-up view of the glycans at N53 (blue sticks) and N90 (yellow sticks) on ACE2. (e) Close-up view of the glycans at N53 (blue sticks), N90 (yellow sticks), N322 (black sticks) and N546 (red sticks). 
We next quantified the complete solvent-accessible surface area (SASA) of the Spike protein in complex with ACE2, both with and without glycans. The average accessible area of protein atoms for non-glycosylated and glycosylated Spike was $1395 \mathrm{~nm}^{2}$ and $864 \mathrm{~nm}^{2}$, respectively, ACE2 or its glycans (Fig. 1b, c, Fig. 2b, c). It has been reported that Spike mutants lacking the
glycans at N331 and N343 display reduced infectivity, while elimination of the glycosylation indicating that glycans shield about $38 \%$ of the protein surface of Spike, a value that is comparable to what was previously found in simulations of Spike alone. ${ }^{23,32}$ The area of protein atoms that are shielded by the individual glycans are shown in Fig. 2a and Fig. S4.

Further analysis showed that glycans at N122, N165 and N343 on Spike directly interact with motif at N234 results in increased resistance to neutralizing antibodies, without reducing infectivity of the virus. ${ }^{33}$ The equilibrium between the "up" and "down" conformations of Spike involves various stabilizing and destabilizing effects, with possible roles for the glycans at N165, N234, N331 and N343. ${ }^{22,26,34}$ Removing the glycans at N165, N234 and N343 was experimentally seen to reduce binding to ACE2 by $10 \%, 40 \%$ and $56 \%$, respectively. ${ }^{22,26}$ In our MD simulations, the glycan at position N343 interacts directly with ACE2 (Fig. 2), while the glycan at N331 interacts with a neighboring Spike monomer (Fig. 1c, Fig. S5), indicating that the N331 glycosylation site only indirectly affects the interaction of Spike with ACE2. In our model, the glycan at N234 also does not interact directly with ACE2, but seems to stabilize the "up" 150 conformation. Its removal could favor the "down" conformation of the RBD, possibly explaining the observed more effective shielding against neutralizing antibodies. In agreement with previous simulations $^{22}$ the Man9 glycan at N234 of Spike partially inserts itself into the vacant space in the core of the trimer that is created when the RBD of monomer 3 is in the "up" conformation (Fig. S6). In our simulations, the free space created by the "up" conformation seems slightly 155 smaller for Spike in complex with ACE2, suggesting that binding to ACE2 has a stabilizing effect on the Spike monomer.

The N165Q mutant was experimentally found to be more sensitive to neutralization. ${ }^{33}$ In our models, the glycan at N165 is positioned directly next to the RBD (Fig. 1b) and thus could shield important antigenic sites. These data highlight the complex impact of Spike glycosylation on the 160 intramolecular interactions of the Spike monomers and, critically, the interaction with ACE2, posing a challenge to design SARS-CoV-2 neutralizing moieties. 

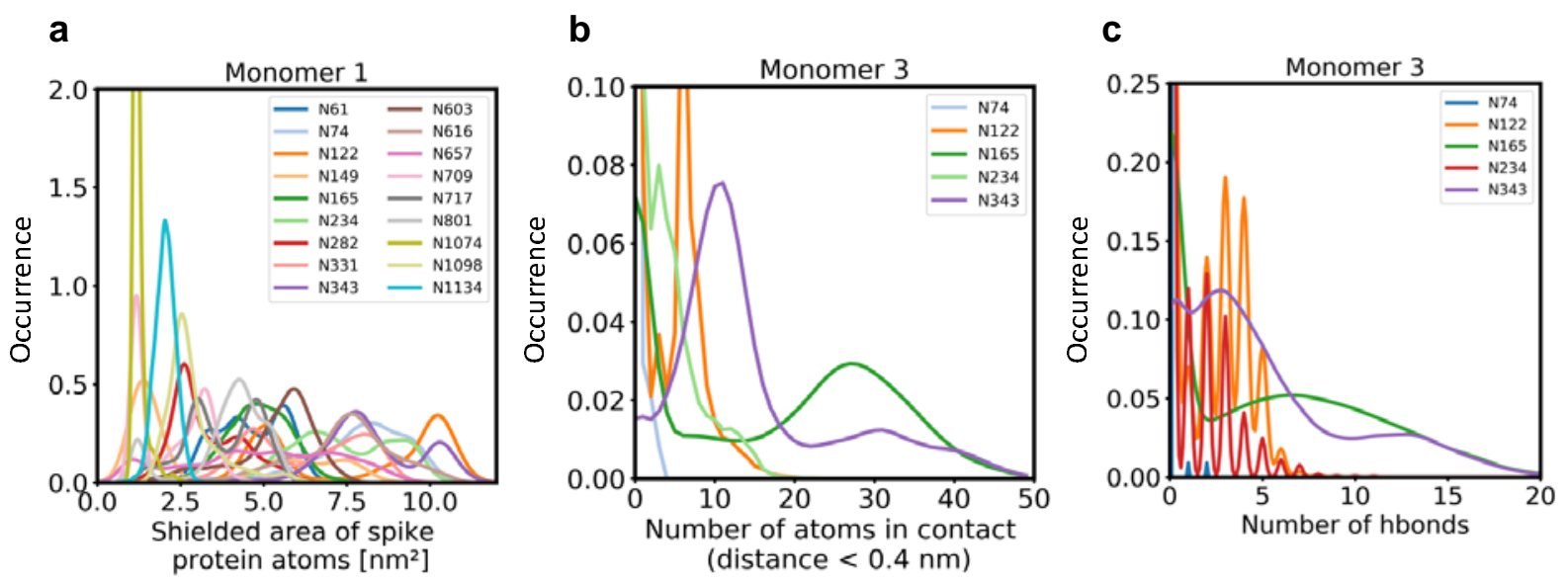

Figure 2. Role of Spike glycosylation in shielding the protein surface and interactions with hACE2. (a) Normalized distribution of the area of Spike protein atoms that is shielded by each of its glycans on monomer 1 (see Fig. S4 for monomers 2 and 3). (b) Normalized distribution of the number of atoms in contact with ACE2 and (c) the number of hydrogen bonds with ACE2 for glycans on monomer 3 of Spike (see Fig. S5 for monomers 1 and 2).

Since our modeling clearly confirmed that ACE2 glycosylation plays a significant role in its binding to Spike (Fig. 1d,e), we also determined the area of the Spike-ACE2 interface region, by subtracting the SASA of the complex from the SASA of the individual proteins and dividing by two. The total interface area was $24.6 \mathrm{~nm}^{2}$, with glycans accounting for up to $51 \%$ of the interface area, i.e. $12.6 \mathrm{~nm}^{2}$, contributed by the four most relevant glycans at positions N53, N90, N322 and N546 of ACE2 (Fig. 3). Furthermore, we scored the number of atoms of each ACE2 glycan in contact with Spike. A contact was defined as a distance of less than $0.4 \mathrm{~nm}$ between two atoms. This allowed us to identify the glycans at N53, N90, N322 and N546 as interacting with Spike, with the glycan at position N53 having the weakest interaction. Notably, N546 interacted with Spike for a significant amount of time only in one of the two independent simulations. The degree of interaction correlated with the spatial proximity between the glycans and the RBD (Fig. 1e, f). Assessing the number of hydrogen bonds that formed during the simulations, the glycans at N90 and N322 appear most prominent (Fig. 3b). Interestingly, the glycans at N90 and N322 interact directly with Spike protein atoms, while the glycan at N546 (red sticks in Fig. 1f) interacts with the glycans at N122 and N165 of Spike (dark green and orange sticks in Fig. 1b). These findings are in agreement with previously reported simulations of the complexes. ${ }^{21,25}$ 

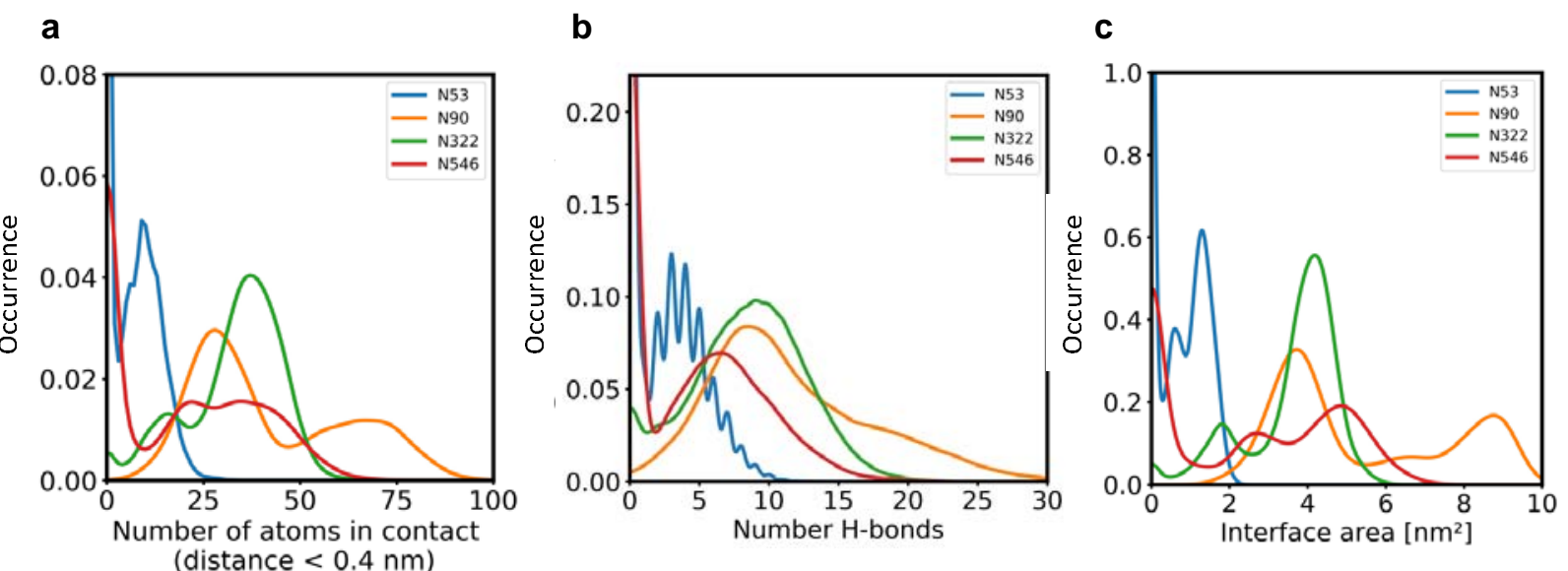

Figure 3. The role of hACE2 glycosylation in the interaction with Spike. (a) Normalized distribution of the number of atoms of glycans at N53, N90, N322 and N546 of ACE2 that are in contact with Spike (distance $<0.4$ $\mathrm{nm}$ ). (b) Normalized distribution of the number of hydrogen bonds between glycans at N53, N90, N322, N546 of ACE2 and Spike. (c) Normalized distribution of the interface area between Spike and glycans at N53, N90, N322 and N546 of ACE2.

Next, we assessed the conformational freedom of ACE2 glycans upon binding to Spike and compared their respective density maps in the simulations of free ACE2, and ACE2 in complex with Spike (Fig. 4). The density map of the unbound ACE2 (Fig. 4a) shows a continuous density of glycans, largely covering the interface area. Formation of the ACE2-Spike complex significantly reduces the conformational freedom of the glycans, in particular the ones at N90 and N322 (Fig. 4b). We predict that the glycans at N90 and N322 hamper binding to Spike, either sterically or through an entropic penalty upon binding due to a loss of conformational freedom. These glycans have been implicated as being relevant for binding before, ${ }^{21}$, as well as 200 the glycan at N53, ${ }^{35}$ but no conclusions were drawn if they contribute positively or negatively to binding. Mehdipour and Hummer predicted the glycan at N322 to contribute favorably to binding, because of the favorable interactions of this glycan with the Spike surface. ${ }^{25}$ We did not observe a significantly more pronounced interaction with Spike for the glycan at N322, compared to the one at N90 (Fig. 3). Based on conformational considerations, we therefore rather predict a negative impact on binding for both glycans (Fig. 4). 
a

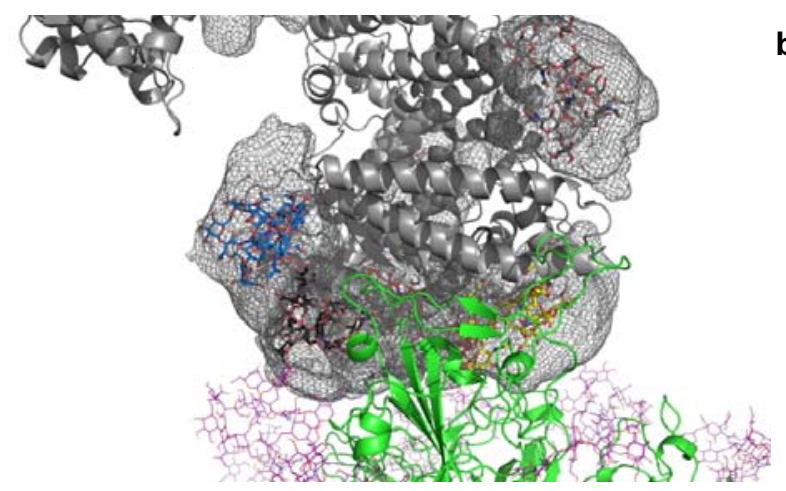

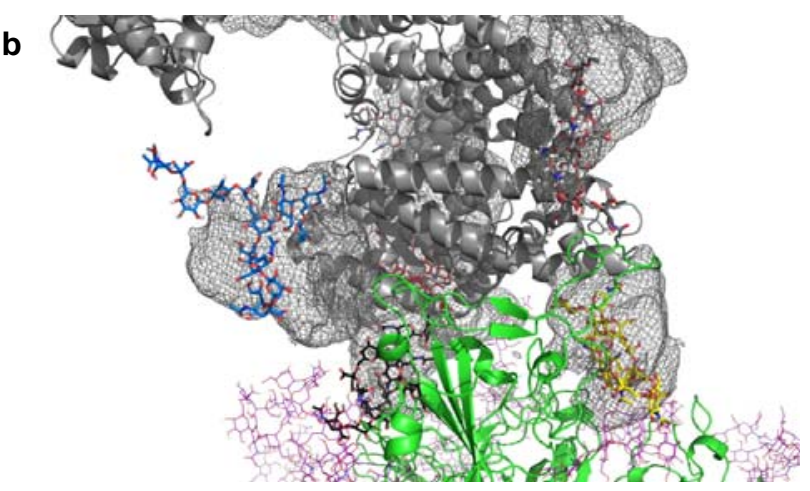

Figure 4. Average location density maps of glycans on ACE2. (a) The density map (grey mesh) of the glycans at N53, N90, N322 and N546 as observed in the simulations of unbound ACE2 are superimposed onto the ACE2 Spike complex. (b) The density map of the same glycans, as observed in the simulation of the ACE2 - Spike complex. ACE2 in grey, Spike in green. Single, randomly selected conformations of the glycans are shown in blue (N53), yellow (N90), black (N322) and red (N546).

Since only the glycans at N90 and N322 directly interact with the protein atoms of the Spike proteins, while the glycan on N546 forms hydrogen bonds with glycans present on Spike, we set out to confirm the negative influence of N90 and N322 glycosylation on the interactions with Spike experimentally. First, we ablated N-glycosylation at N90 and N322 individually using the ACE2-Fc fusion constructs ACE2-T92Q-Fc (ref. ${ }^{15}$ ) and ACE2-N322Q-Fc. Note that ref. ${ }^{15}$ indeed suggests that removal of the glycan at N90 through a mutation of T92 leads to enhanced interaction with Spike. The same data set, however, suggests that removal of the glycan at N322 through a mutation of T324 most likely leads to reduced affinity to Spike. However, T324 is itself part of the interface with Spike (Fig. S7), and any mutation of this residue could easily disrupt ACE2 - Spike binding directly, rather than through its effect on the N322 glycosite. We therefore decided to mutate N322 into glutamine to prevent glycosylation at this position.

The wild-type and mutant ACE2-Fc constructs were expressed in HEK293-6E cells and purified from the culture supernatants by protein A affinity chromatography to apparent homogeneity (Fig. S8). Analysis by size-exclusion chromatography combined with detection by multi-angle light scattering (SEC-MALS) demonstrated that all purified proteins were dimers of the expected native molecular mass (Fig. S9). The impact of the introduced mutations on the overall fold of ACE2-Fc was tested with differential scanning calorimetry (DSC), a sensitive biophysical 230 method for the assessment of the thermal stability of proteins. Three thermal transitions could be discriminated. The first midpoint of transition $\left(T_{\mathrm{m}} 1\right)$ is due to the unfolding of ACE2, whereas 
the second and third midpoints of transitions ( $T_{\mathrm{m}} 2$ and $\left.T_{\mathrm{m}} 3\right)$ reflect the thermal denaturation of the $\mathrm{C}_{\mathrm{H}} 2$ and $\mathrm{C}_{\mathrm{H}} 3$ domains of the $\mathrm{Fc}$ part of the fusion proteins. ${ }^{36}$ The $T_{\mathrm{m}} 1$ midpoint transition temperatures of the ACE2-Fc glycomutants $\left(53.3-54.0^{\circ} \mathrm{C}\right)$ were slightly higher than for the wildtype protein $\left(52.2^{\circ} \mathrm{C}\right)$, while $T_{\mathrm{m}} 2$ and $T_{\mathrm{m}} 3$ remained unchanged (Fig. 5). This indicates that removal of the N90 and N322 glycans does not compromise the structural integrity of ACE2.
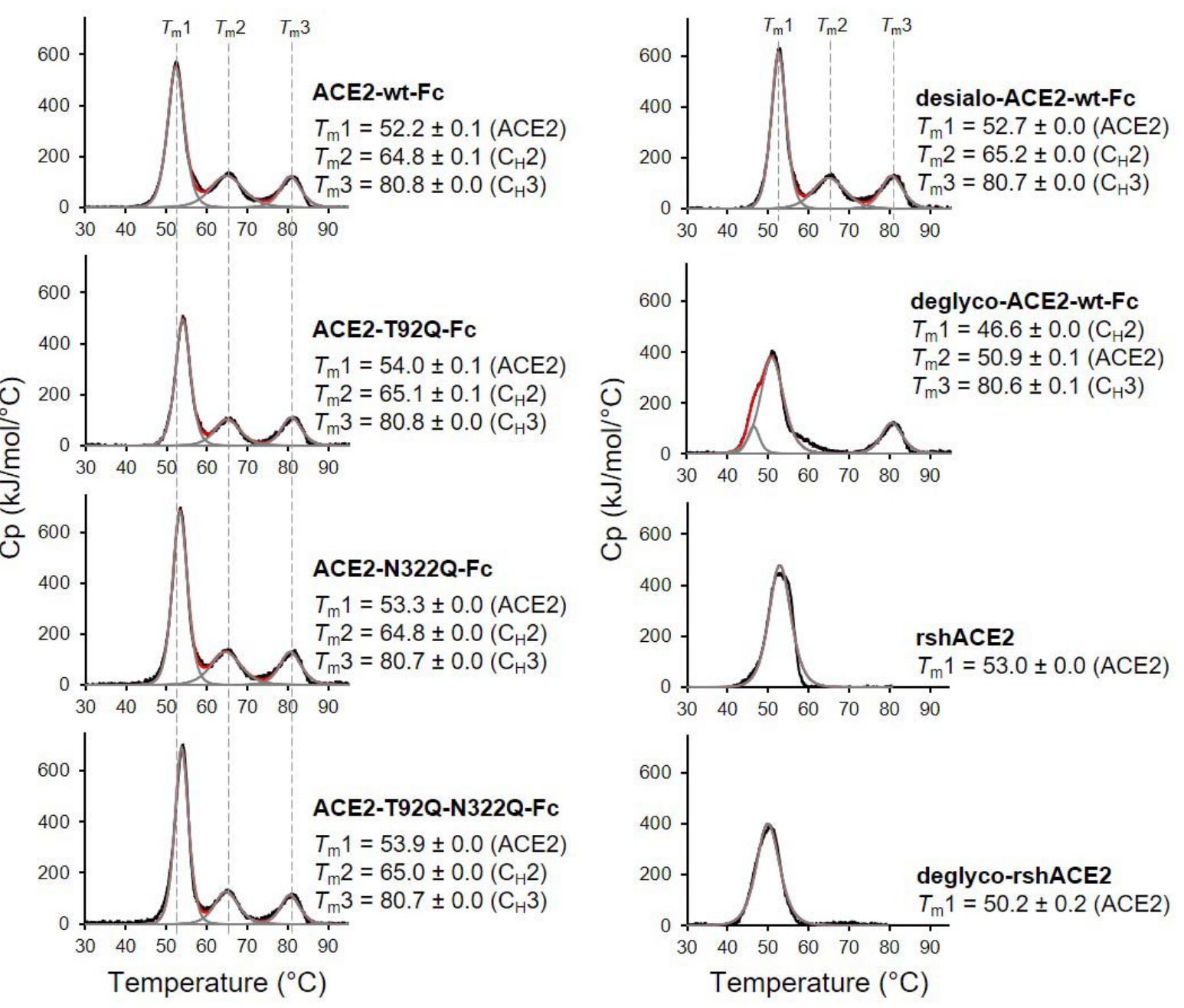

Figure 5. Analysis of ACE2 variants by differential scanning calorimetry (DSC). Raw data (black) were smoothened (red) and then fitted using a non-two-state thermal unfolding model (grey). Data are presented as mean $240 \pm$ SEM of three independent experiments. Cp, heat capacitance; rshACE2, clinical-grade recombinant soluble human ACE2; deglyco-rshACE2, enzymatically deglycosylated rshACE2; deglyco-ACE2-wt-Fc, enzymatically deglycosylated wild-type ACE2-Fc; desialo-ACE2-wt-Fc, enzymatically desialylated wild-type ACE2-Fc.

The Spike-binding properties of the purified ACE2-Fc variants were characterized by biolayer interferometry (BLI). For this, ACE2-wt-Fc, ACE2-T92Q-Fc and ACE2-N322Q-Fc were 
biotinylated, immobilized on streptavidin biosensor tips and dipped into serial dilutions of trimeric Spike. Since we did not observe appreciable dissociation of ACE2-Fc/trimeric Spike complexes in our analyses (Fig. S10), we evaluated the association rates ( $k_{\text {obs }}$; Fig. 6a). To determine equilibrium affinity constants $\left(K_{\mathrm{D}}\right)$, we analyzed the interactions between the immobilized ACE2-Fc constructs and monomeric RBD (Fig. 6b, Fig. S11). The BLI data are in good agreement with our computational models, confirming that the removal of protein $\mathrm{N}$ glycosylation at either $\mathrm{N} 90$ or N322 results in up to 2-fold higher binding affinities, when compared to ACE2-wt-Fc (ACE2-wt-Fc: $K_{\mathrm{D}}=16.2 \pm 0.7 \mathrm{nM}$; ACE2-T92Q-Fc: $K_{\mathrm{D}}=8.0 \pm 0.7$ nM; ACE2-N322Q-Fc: $K_{\mathrm{D}}=11.4 \pm 0.3 \mathrm{nM}$; Fig. 6b; Fig. S11). Thus, structure-guided glycoengineering at N90 and N322 results in ACE2 forms with increased affinity for SARS-CoV-2 Spike binding.
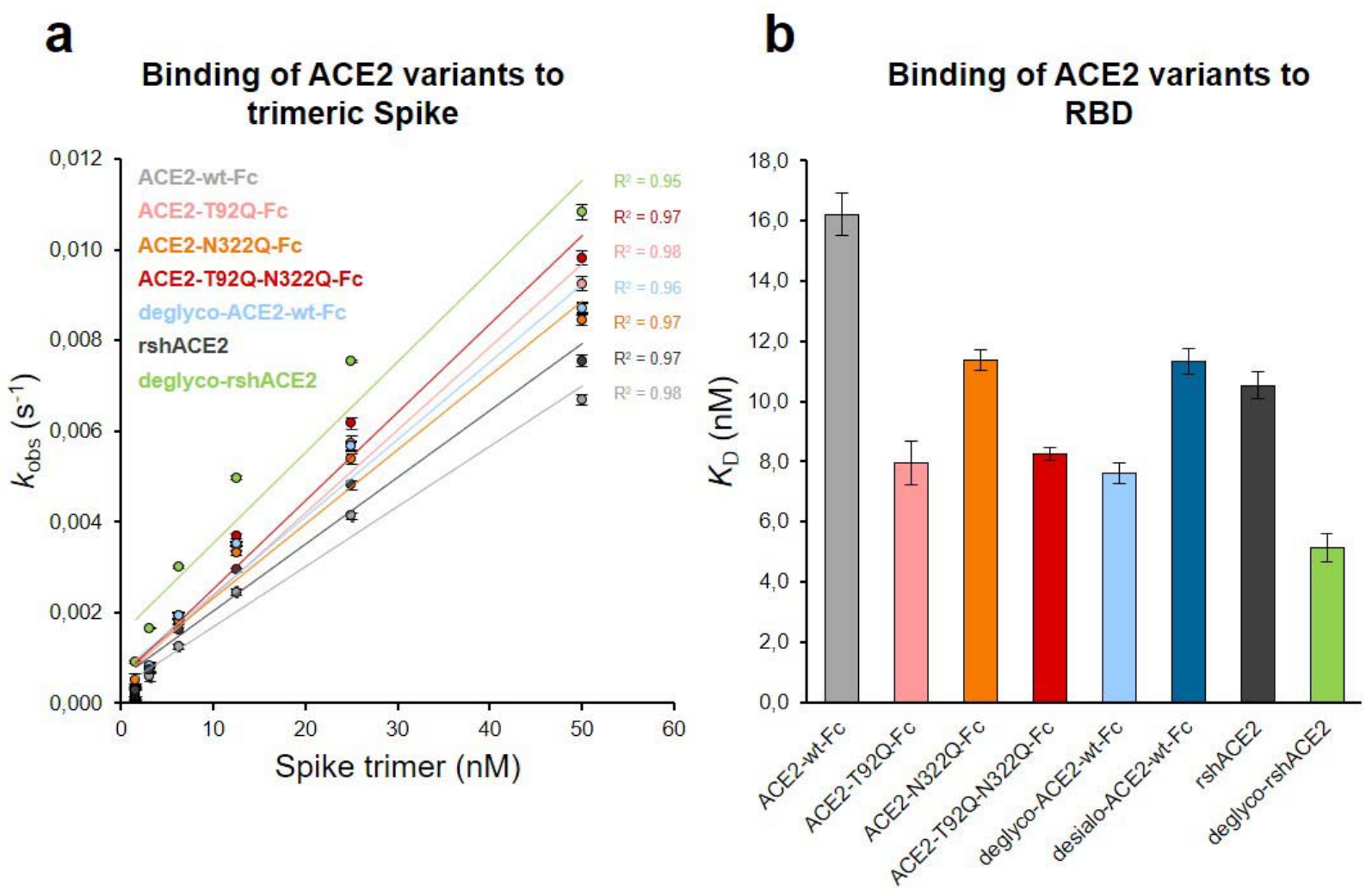

Figure 6. Binding of Spike and RBD to glyco-engineered ACE2 variants. (a) Binding of Spike to glycoengineered ACE2 variants as determined by biolayer interferometry (BLI). Plots of $k_{\mathrm{obs}}$ (observed association rate) as a function of Spike concentration were generated by fitting the association data to a 1:1 binding model. Binding analysis was performed by dipping ACE2-loaded biosensors into 2-fold serial dilutions of purified Spike (1.6-50 $\mathrm{nM})$. All measurements were performed in triplicates. rshACE2, clinical-grade recombinant soluble human ACE2; deglyco-rshACE2, enzymatically deglycosylated rshACE2; deglyco-ACE2-wt-Fc, enzymatically deglycosylated 
wild-type ACE2-Fc. (b) $K_{\mathrm{D}}$ values for the interaction of the indicated glyco-engineered ACE2 variants with monomeric RBD. Data are presented as mean \pm SEM of 3 independent experiments. Desialo-ACE2-wt-Fc, enzymatically desialylated wild-type ACE2-Fc.

Next, we tested the virus neutralization properties of ACE2-wt-Fc, ACE2-T92Q-Fc and ACE2N322Q-Fc. For this, we infected Vero E6 cells with 60 plaque-forming units (PFU; multiplicity of infection (MOI): 0.002) of SARS-CoV-2 in the presence of 10-50 $\mu \mathrm{g} / \mathrm{mL}$ ACE2-wt-Fc, ACE2-T92Q-Fc, or ACE2-N322Q-Fc. The extent of SARS-CoV-2 infection and replication was quantified by RT-qPCR detection of viral RNA present in the culture supernatants. Untreated SARS-CoV-2 infected cells released up to 10 times more viral RNA than ACE2-wt-Fc treated cells. Importantly, co-incubation of cells with SARS-CoV-2 and ACE2-T92Q-Fc resulted in significant further reduction of the viral load when compared to ACE2-wt-Fc. Enhanced SARSCoV-2 neutralization was also observed for ACE2-N322Q-Fc. However, this mutant was less effective in promoting virus neutralization than ACE2-T92Q-Fc (Figs. 7 and S12). Similar results were obtained when SARS-CoV-2 neutralization assays were performed with much larger amounts of inoculated virus (MOI: 20) and concomitantly increased ACE2-Fc concentrations (Fig. S13). Hence, in line with our structural glycan interaction map, the removal of either of the N-glycans attached to N90 and N322 gives rise to ACE2 decoy receptors with improved SARSCoV-2 neutralization properties.

$10 \mu \mathrm{g} / \mathrm{mL}$

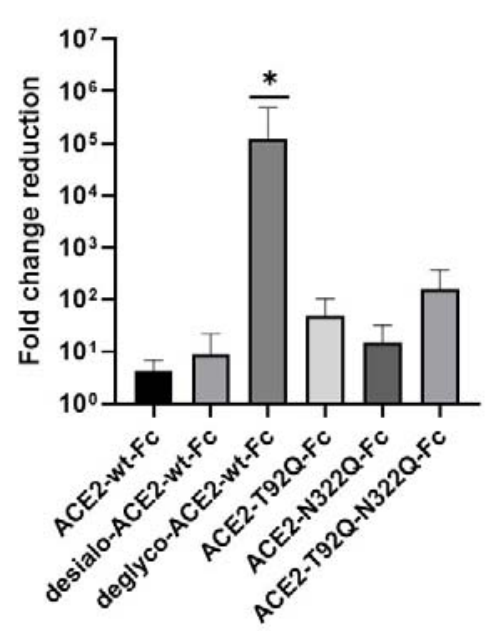

$20 \mu \mathrm{g} / \mathrm{mL}$

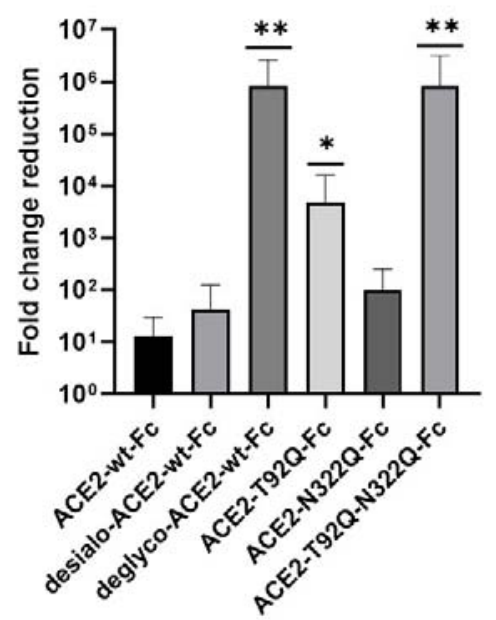

$50 \mu \mathrm{g} / \mathrm{mL}$

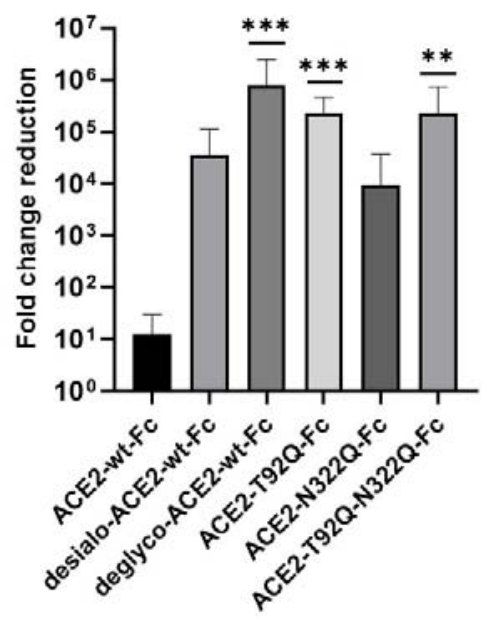


Figure 7. Critical role of ACE2 glycosylation for SARS-CoV-2 infectivity. Inhibition of SARS-CoV-2 infection of Vero E6 cells using wild-type ACE2-Fc and the indicated glyco-engineered ACE2-Fc variants at final concentrations of $10-50 \mu \mathrm{g} / \mathrm{mL}$. The viral RNA content of the culture supernatants was quantified by RT-qPCR and expressed as fold change reduction relative to untreated controls. Data are presented as mean \pm SEM of 2-3 independent experiments each performed in triplicates. Deglyco-ACE2-wt-Fc, enzymatically deglycosylated wildtype ACE2-Fc; desialo-ACE2-wt-Fc, desialylated wild-type ACE2-Fc. ${ }^{*} \mathrm{P}<0.05 ; * * \mathrm{P}<0.01 ; * * * \mathrm{P}<0.001$ (KruskalWallis).

To investigate a potential additive effect of simultaneous elimination of N-glycosylation at N90 and N322, we generated a double mutant ACE2-T92Q-N322Q-Fc construct. We also digested ACE2-wt-Fc with peptide-N4-(N-acetyl-beta-glucosaminyl)asparagine amidase F (PNGase F) to remove all accessible $\mathrm{N}$-glycans (deglyco-ACE2-wt-Fc) and neuraminidase to release terminal sialic acid residues (desialo-ACE2-wt-Fc). Purity and homogeneity of these additional ACE2-Fc variants was ascertained by SDS-PAGE and SEC-MALS (Figs. S8 and S9). The absence of Nglycans attached to N90 and/or N322 in ACE2-T92Q-N322Q-Fc and the respective single mutants was demonstrated by LC-ESI-MS (Fig. S14). Quantitative release of sialic acids and complete removal of $\mathrm{N}$-glycans from all ACE2-wt-Fc N-glycosylation sites with the exception of N546 was also confirmed (Figs. S15 and S16). The glycans at N546 of ACE2-wt-Fc exhibited partial resistance $(40 \%)$ to PNGase F treatment (Fig. S15). Combined introduction of the mutations T92Q and N322Q as well as enzymatic desialylation did not reduce the thermal 305 stability of ACE2-Fc as assessed by DSC, while close-to-complete removal of N-glycans by PNGase F led to a slightly decreased midpoint transition temperature of the ACE2 domain (Fig. 5). Studies of the interaction between ACE2-T92Q-N322Q-Fc and deglyco-ACE2-wt-Fc with RBD by BLI analysis yielded $K_{\mathrm{D}}$ values similar to those determined for the single mutant ACE2T92Q-Fc (ACE2-T92Q-N322Q-Fc: $K_{D}=8.2 \pm 0.2 \mathrm{nM}$; deglyco-ACE2-wt-Fc: $K_{\mathrm{D}}=7.6 \pm 0.3$

$310 \mathrm{nM})$. The affinity of desialo-ACE2-wt-Fc for RBD $\left(K_{\mathrm{D}}=11.3 \pm 0.4 \mathrm{nM}\right)$ was also higher than that of native ACE2-wt-Fc (Fig. 6b). The increased affinities of these ACE2-Fc variants for Spike correlate well with their potencies to neutralize SARS-CoV-2, with deglyco-ACE2-wt-Fc followed by ACE2-T92Q-N322Q-Fc displaying the highest neutralization potencies (Figs. 7 and S13). The effect of desialo-ACE2-wt-Fc on SARS-CoV-2 infections of Vero E6 cells was less 315 pronounced and comparable to that of the single mutant ACE2-N322Q-Fc (Fig. 7), in good agreement with the almost identical RBD-binding affinities of these two ACE2-Fc variants (Fig. 
6b). Taken together, these data identify critical glycans at position N90 and N322 of ACE2 that structurally and functionally interfere with Spike-ACE2 binding; ablation of these glycans via site-directed mutagenesis or enzymatic deglycosylation generated ACE2 variants with improved Spike-binding properties and increased neutralization strength.

The results presented above uncover the critical importance of N-glycans located at the ACE2Spike interface for the infection of host cells by SARS-CoV-2. This prompted us to test the feasibility of removing all N-glycans from clinical-grade rshACE2, which has undergone placebo-controlled phase 2 clinical testing in 178 COVID-19 patients (ClinicalTrials.gov 325 Identifier: NCT04335136), and to test for its SARS-CoV-2 neutralization properties. To this end, we generated enzymatically deglycosylated clinical-grade rshACE2 (deglyco-rshACE2) using PNGase F. The quantitative release of all N-glycans, with the exceptions of those attached to the N432 and N546 glycosites, was confirmed by LC-ESI-MS/MS (Fig. S15), and the integrity and homogeneity of dimeric deglyco-rshACE2 was demonstrated by SEC-MALS (Fig. S9). 330 Paralleling our observations with deglyco-ACE2-wt-Fc, we found the binding affinity of deglyco-rshACE2 to $\operatorname{RBD}\left(K_{\mathrm{D}}=5.1 \pm 0.5 \mathrm{nM}\right)$ to be two times higher than for native rshACE2 $\left(K_{\mathrm{D}}=10.5 \pm 0.4 \mathrm{nM}\right.$; Fig. 6b). Furthermore, deglyco-rshACE2 displayed improved SARS-CoV2 neutralization properties in Vero E6 cell infection assays. At a final concentration of 200 $\mu \mathrm{g} / \mathrm{mL}$ deglyco-rshACE2, we observed a significant reduction in SARS-CoV-2 replication when compared to treatment with the native form of the protein (Fig. 8). 
a

\section{$200 \mu \mathrm{g} / \mathrm{mL}$}

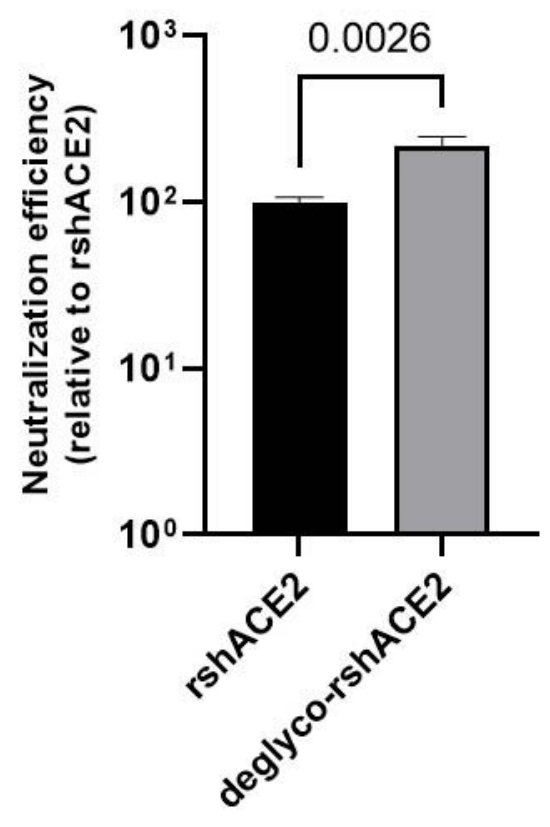

b

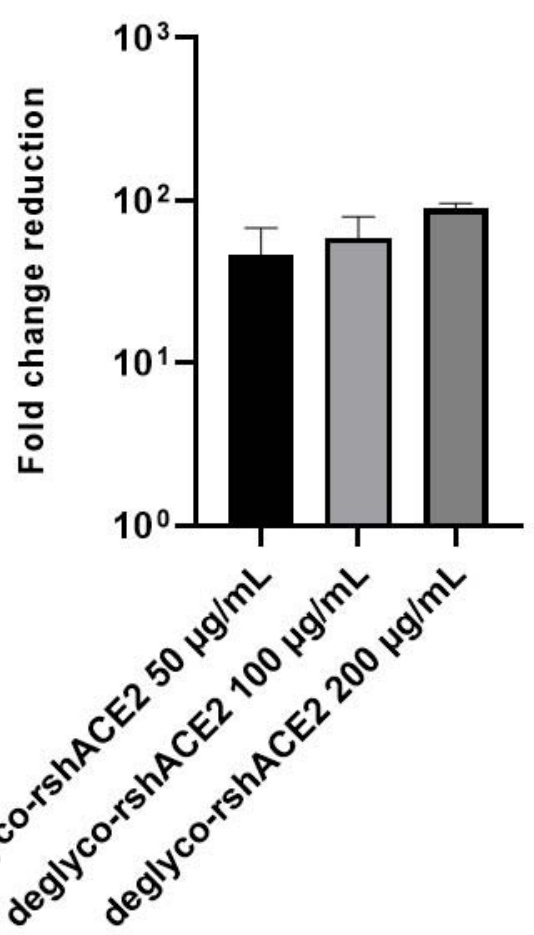

Figure 8. Deglycosylated rshACE2 is a potent SARS-CoV-2 decoy receptor. (a) Inhibition of SARS-CoV-2 infection of Vero E6 cells at an MOI of 20 using native and enzymatically deglycosylated rshACE2 at final concentrations of $200 \mu \mathrm{g} / \mathrm{mL}$. The viral RNA content of the infected cells was quantified by RT-qPCR and expressed as neutralization efficiency relative to native rshACE2 (set to 100\%). Data are presented as mean \pm SEM of 4 independent experiments. Deglyco-rshACE2, enzymatically deglycosylated rshACE2; $\mathrm{P}=0.0026$ (Student's $t$ test). (b) Inhibition of SARS-CoV-2 infection of Vero E6 cells at an MOI of 20 using deglyco-rshACE2 at final concentrations of 50-200 $\mu \mathrm{g} / \mathrm{mL}$. The viral RNA content of the infected cells was quantified by RT-qPCR and expressed as fold change reduction relative to untreated controls. Data are presented as mean \pm SD of triplicates.

Besides serving as a soluble decoy receptor to prevent SARS-CoV-2 infection of ACE2expressing host cells, rshACE2 also regulates blood pressure and protects multiple organs such as the heart, kidney and lung as well as blood vessels via enzymatic degradation of angiotensin II. ${ }^{37}$ In contrast to other recently described ACE2 mutants displaying improved Spike binding concomitant with inadvertently or intentionally impaired enzymatic activity, ${ }^{14,15}$ the catalytic 
activities of ACE2-T92Q-Fc, ACE2-N322Q-Fc and ACE2-T92Q-N322Q-Fc were found to be only modestly reduced as compared to ACE2-wt-Fc (ACE2-T92Q-Fc: $65 \pm 11 \%$; ACE2N322Q-Fc: $69 \pm 7 \%$; ACE2-T92Q-N322Q-Fc: $79 \pm 11 \%$; Fig. 9).

ACE2-wt-Fc

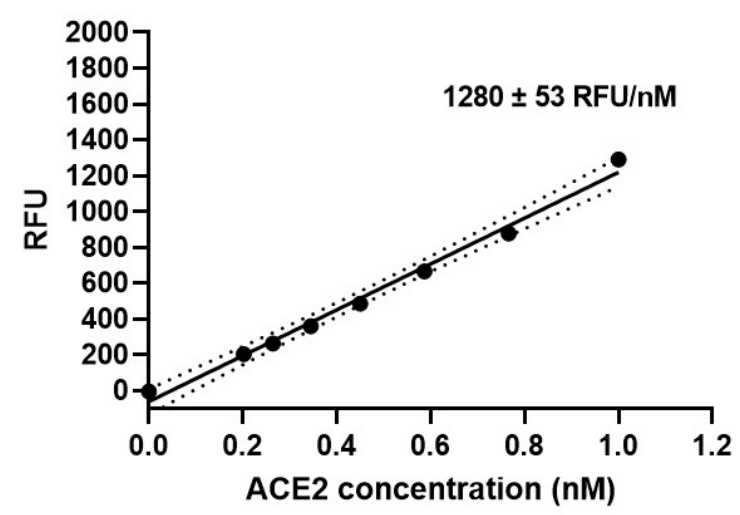

ACE2-T92Q-FC

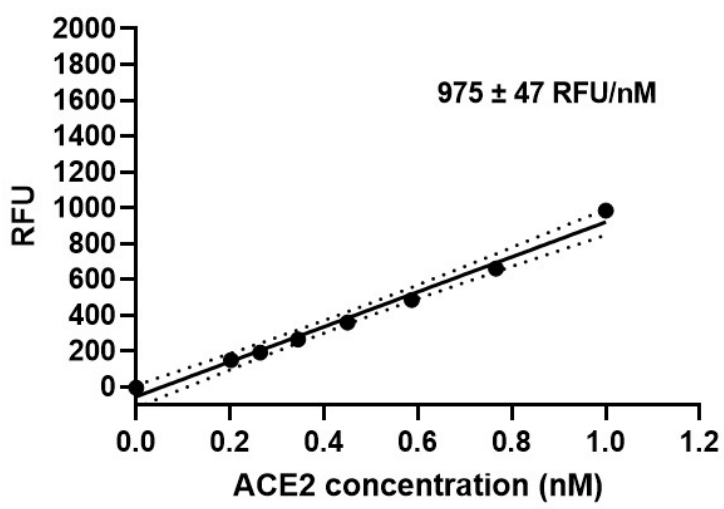

ACE2-T92Q-N322Q-Fc

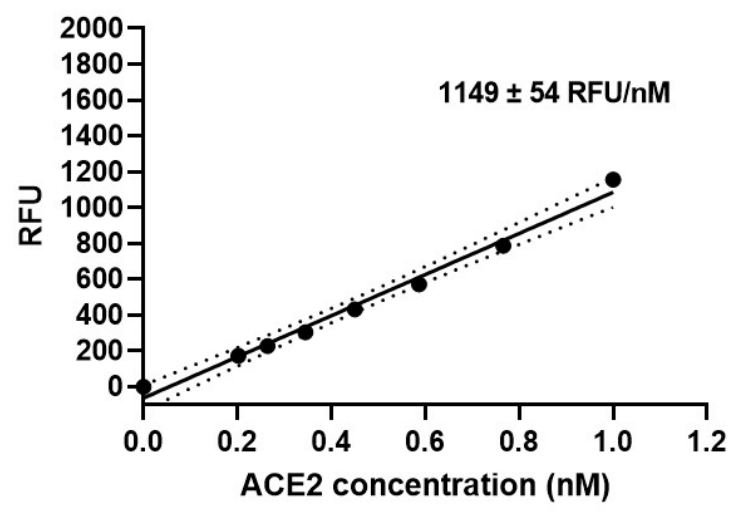

ACE2-N322Q-FC

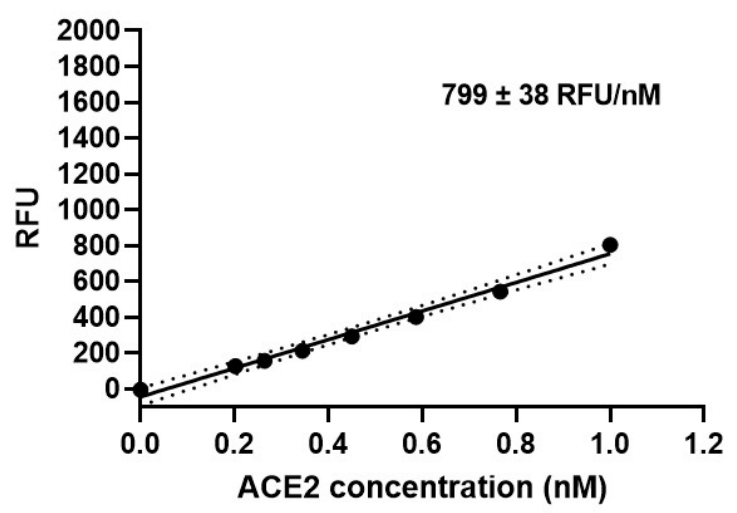

Figure 9. Enzymatic activity of ACE2-Fc mutants. Hydrolysis of $100 \mu \mathrm{M}$ 7-methoxycoumarin-4-yl-acetyl-AlaPro-Lys-2,4-dinitrophenyl was continuously monitored by spectrofluorimetry. Hydrolytic activity is plotted as relative fluorescence units (RFU) over ACE2-Fc concentration (in $\mathrm{nM}$ ). All assays were performed in technical triplicates. One representative experiment out of two is shown.

Interestingly, deglyco-ACE2-wt-Fc (149 $\pm 1 \%)$ and desialo-ACE2-wt-Fc (160 $\pm 2 \%)$ exhibited higher enzymatic activities than native ACE2-wt-Fc (Figs. 10 and S17). A similar observation was made for deglyco-rshACE2, although the enhancing effects of enzymatic deglycosylation on catalytic efficiency were less pronounced $(113 \pm 2 \%$ as compared to native rshACE2; Fig. 10). These results show that enzymatic removal of N-glycans from ACE2-Fc and clinical-grade 
rshACE2 results in increased Spike binding and enhanced SARS-CoV-2 neutralization while preserving its potentially critical enzymatic activity.

ACE2-wt-Fc

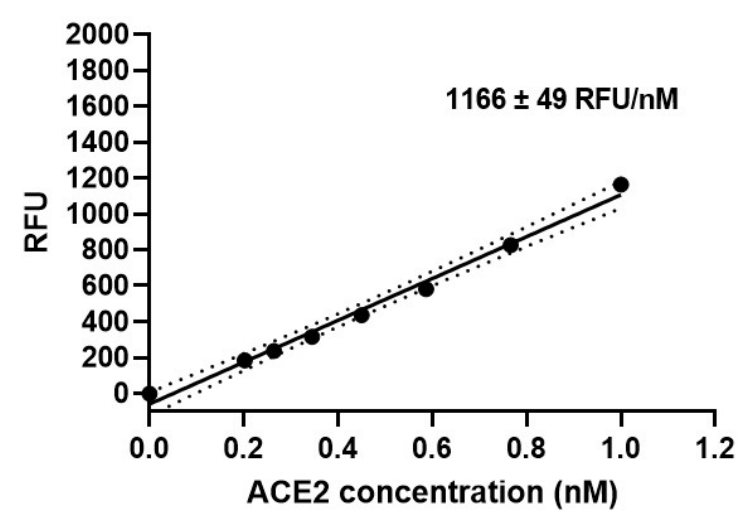

rShACE2

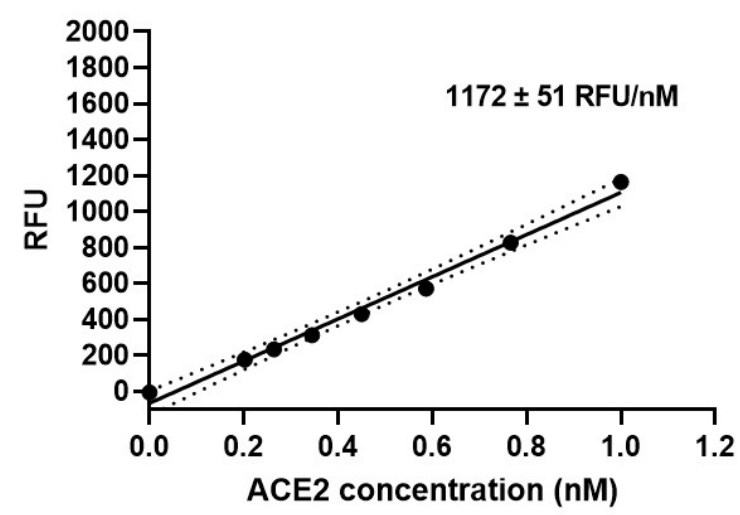

deglyco-ACE2-wt-Fc

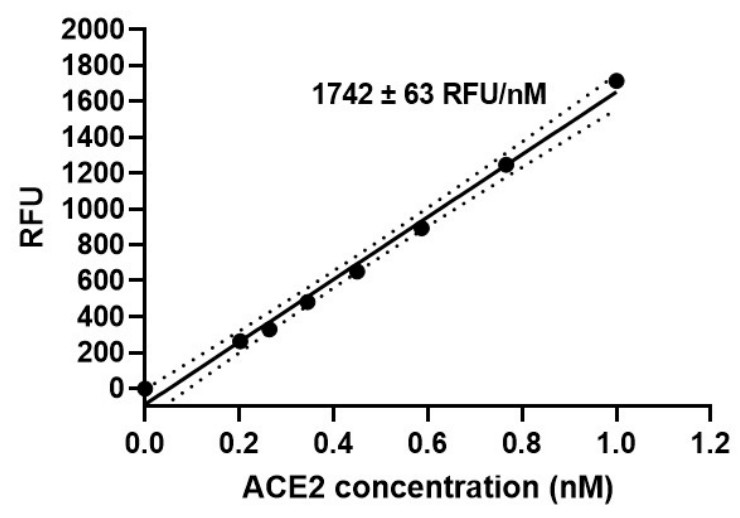

deglyco-rsh-ACE2

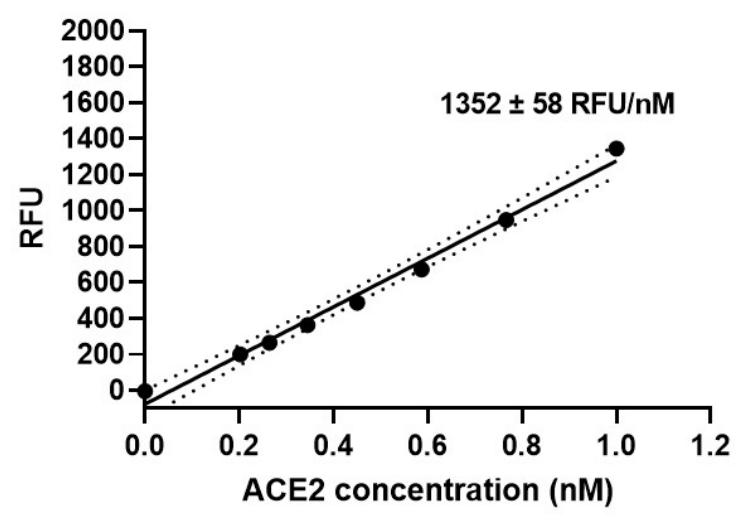

Figure 10. Enzymatic activity of enzymatically deglycosylated ACE2. Hydrolysis of $100 \mu \mathrm{M}$ 7methoxycoumarin-4-yl-acetyl-Ala-Pro-Lys-2,4-dinitrophenyl was continuously monitored by spectrofluorimetry. Hydrolytic activity is plotted as relative fluorescence units (RFU) over ACE2 concentration (in nM). All assays were performed in technical triplicates. One representative experiment out of two is shown.

\section{Discussion}

375 Our data demonstrate that structure-guided glycoengineering is a powerful means to develop ACE2 variants with improved SARS-CoV-2 neutralization properties without compromising the structural stability and catalytic activity of the enzyme. Our in silico models of the Spike-ACE2 complex combined with simulations of its spatial and temporal dynamics rationalized previously published data and led to predictions that were confirmed by in vitro binding studies and cell- 
based SARS-CoV-2 neutralization assays. It is of note that the moderately enhanced affinity of ACE2 glycovariants for monomeric RBD observed in biolayer interferometry experiments relates to a far more pronounced increase of their virus-neutralization potency. This may be explained by multiple cooperative effects. First, a cooperative effect may be expected for the association of trimeric Spike molecules present in the viral envelope with membrane-bound ACE2 dimers. In this supramolecular setting, a subtle increase in the affinity of ACE2 for RBD can lead to a dynamic equilibrium of binding and unbinding events with up to six potential interactions, leading to an overall much stronger avidity effect. Second, a slight advantage of the soluble ACE2 decoy receptor over endogenous native ACE2 may be sufficient to tip the balance between SARS-CoV-2 attachment and shedding of viral particles from the host cell surface. Third, blocking of initial binding prevents viral propagation and hence spread of the virus to surrounding cells. Finally, it is possible that the N-glycan moiety of ACE2 also modulates other aspects of viral entry besides promoting the docking of Spike to the cell surface. ${ }^{29}$

It has been reported that the sialylation status of ACE2 affects its interactions with SARS-CoV-2 Spike. ${ }^{30}$ We have found that enzymatic desialylation of ACE2 results in a reproducible increase 395 of its affinity to RBD without detectable structural penalties. Importantly, desialylated ACE2 is more efficient in neutralizing SARS-CoV-2 than its native counterpart. Molecular simulations suggest that the terminal sialic acids of the N-glycans attached to ACE2 residues N90 and N322 mask parts of the Spike-ACE2 interface and thus could interfere with Spike binding through steric clashes and/or electrostatic effects (Fig. S18). This provides a structural rationale how sialic acids present on ACE2 might dampen interactions with Spike during SARS-CoV-2 attachment to host cells. ${ }^{38}$

In line with other reports, ${ }^{15,38}$ our results indicate that the elimination of the N-glycans attached to N90 is largely responsible for the improved Spike-binding properties of enzymatically deglycosylated ACE2. As proposed ${ }^{14,15}$ and corroborated by our mutational analysis, substitution of ACE2 residues N90 or T92 could indeed provide an alternative approach for the development of ACE2 variants with improved SARS-CoV-2 sequestering properties. Our data indicate that ablation of N90 glycosylation could be combined with mutations of N322 and possibly other ACE2 N-glycosylation sites to achieve an even higher SARS-CoV-2 neutralizing potency. However, expression of an ACE2 variant lacking all potential N-glycosylation sites in ACE2410 negative host cells led to reduced rather than enhanced susceptibility of the cells to SARS-CoV-2 
as compared to transduction with wild-type ACE2. ${ }^{38}$ This was attributed to the much lower cellular content of the mutant protein relative to the native enzyme, thus demonstrating that the importance of $\mathrm{N}$-glycosylation for proper folding of glycoproteins during their biosynthesis ${ }^{39}$ also applies to ACE2. Given the inferior expression yields of glycan-free ACE2 and the potential of unwanted immunological side effects when non-natural mutations are introduced into a therapeutic glycoprotein, we believe that the clinical potential of enzymatically deglycosylated rshACE2 is superior to that of any of our ACE2 glycomutants. In our opinion, treatment of clinical-grade rshACE2 with deglycosylation enzymes such as PNGase F followed by a final polishing step represents a straightforward, Good Manufacturing Practice (GMP)-compliant and 420 industrially feasible alternative to generate a potent therapeutic drug for the treatment of SARSCoV-2 infected persons and patients. 


\section{Materials and Methods}

\section{Modeling of the Spike-hACE2 complex}

To model the fully glycosylated SARS-CoV-2 Spike-human ACE2 (hACE2) complex, a protein model was created using partial experimental structures deposited in the protein databank (PDB). The Spike RBD domain in complex with hACE2 ${ }^{17}$ (PDB: 6M17) was superimposed with the opened RBD domain in a Spike structure with one open RBD domain ${ }^{18}$ (PDB: 6VYB). Alternative Spike structures have been published, which show very similar conformations. ${ }^{19}$ Similarly, further structures of the Spike RBD-hACE2 complex ${ }^{4,16}$ have been reported which show very similar conformations to the templates used. Missing residues in Spike were modeled using SWISS-MODEL ${ }^{40}$ and the superimposed structure as template based on the complete SARS-CoV-2 S sequence (GenBank QHD43416.1).

Different types of glycans were added to Spike and hACE2. For Spike, the assignments of Watanabe et al. ${ }^{20}$ were followed, selecting oligomannosidic (Man5 or Man9) or complex (biantennary di-sialylated core fucosylated; $\mathrm{NaNaF}$ ) glycans according to the majority of the glycans detected at the respective site. This was largely confirmed by our own analysis. ${ }^{10}$ For hACE2, complex (i.e. bi-antennary di-sialylated core fucosylated) N-glycans were added. See Table S1 for the exact assignments. Initial conformations of the glycans were selected following previously derived procedures. ${ }^{41}$ In brief, molecular dynamics simulations were performed of mini-peptides with the glycans attached. Local Elevation ${ }^{42}$ was used to enhance the sampling of all glycosidic linkages, during simulations of $100 \mathrm{~ns}$. The entire glycan trees were clustered based on the conformations of the individual glycosidic linkages. ${ }^{43}$ This resulted in conformational bundles containing 1301, 1340 and 2413 distinct conformations of Man5, Man9 and $\mathrm{NaNaF}$, respectively. These conformations were fitted onto the respective glycosylation site in the Spike-hACE2 complex using a superposition of the backbone of the asparagine residues and the non-bonded interaction energy between the glycan and protein atoms or previously added glycans was computed. The lowest energy conformation was retained. Topologies and 450 initial conformations were generated using the gromos++ suite of pre- and post-MD tools. ${ }^{44}$ Glycans were added to the complex sequentially, to avoid collisions between individual glycans. A few modeled glycans were incompatible with loops of the Spike protein not resolved in the experimental structures. Loops involved in these structural incompatibilities (residues 141-165 
and 471-490) were partially re-modeled in the fully glycosylated model using the RCD+ loop modeling server. ${ }^{45,46}$ The final model was energy-minimized with the GROMOS 54A8 protein force-field, ${ }^{47,48}$ the GROMOS 53A6glyc glycan force-field ${ }^{41,49,50}$ and the GROMOS simulation software using the steepest decent algorithm. ${ }^{51}$

\section{Molecular dynamics simulations}

Molecular dynamics simulations were performed using the simulation package Gromacs (Version 2019.5) and the indicated force field parameters. hACE2 was reduced to residues 21 to 730 in the models, to reduce its overall size prior to simulation. The models were placed in rhombic dodecahedron simulation boxes and solvated by explicit SPC water molecules. ${ }^{52}$ This resulted in simulation systems of $5.9 \times 10^{5}$ and $2.2 \times 10^{6}$ atoms for hACE2 and the Spike-hACE2 complex, respectively. Two independent 100-ns molecular dynamics simulations were performed for hACE2 and for the Spike-hACE2 complex each. The equations of motion were integrated using a leapfrog integration scheme ${ }^{53}$ with a time-step of 2 fs. Non-bonded interactions were calculated within a cutoff sphere of $1.4 \mathrm{~nm}$ and electrostatic interactions were computed using a particle-particle particle-mesh (P3M) approach. ${ }^{54}$ Bond-lengths were constrained to their optimal values using the Lincs algorithm. ${ }^{55}$ Temperature was maintained at a constant value using a velocity-rescaling algorithm ${ }^{56,57}$ with a relaxation time of 0.1 ps. Pressure was maintained constant using a Parrinello-Rahman barostat ${ }^{58,59}$ with a relaxation time of 2.0 ps and an estimated isothermal compressibility of $4.5 \times 10^{-5} \mathrm{bar}^{-1}$. Configurations were stored every 10 ps for subsequent analyses. Hydrogen bonds were identified using a geometric criterion. A hydrogen bond was logged if the donor-acceptor distance is within $0.25 \mathrm{~nm}$ and the donor475 hydrogen-acceptor angle was larger than 135 degrees. The solvent-accessible surface area was determined by rolling a probe with diameter $0.14 \mathrm{~nm}$ over the surface of the protein, using slices of $0.005 \mathrm{~nm}$ width. An atom contact was assigned if the distance between two atoms were within $0.4 \mathrm{~nm}$. The distributions of atom contacts, hydrogen bonds and solvent-accessible surface area were estimated using a kernel density estimator with gaussian kernels. Distributions obtained 480 from the first and second half of the simulations were compared to ensure convergence. Glycan densities were calculated using the program GROmaps. ${ }^{60}$ 


\section{Recombinant expression of proteins}

Soluble recombinant human ACE2 (rshACE2) was provided by Apeiron Biologicals (Vienna, Austria). Recombinant expression of all other proteins was performed by transient transfection of HEK293-6E cells, licensed from National Research Council (NRC) of Canada, as previously described. ${ }^{36,61}$ Cells were cultivated in FreeStyle F17 expression medium supplemented with $0.1 \%$ (v/v) Pluronic F-68 and 4 mM L-glutamine (all from Thermo Fisher Scientific, United 495 Icahn School of Medicine at Mount Sinai (New York, United States). ${ }^{62,63}$ Plasmid constructs pcDNA3-sACE2(WT)-Fc(IgG1) and pcDNA3-sACE2-T92Q-Fc(IgG1) were obtained from Addgene (United States). The N322Q mutation was introduced into ACE2-wt-Fc and ACE2T92Q-Fc using the QuikChange Lightning Site-Directed-Mutagenesis kit (Agilent Technologies, United States) according to the manufacturer's instructions and the respective parental vector as template. High quality plasmid preparations for expression of ACE2-Fc variants were prepared using the PureYield Plasmid Midiprep System (Promega, United States). Transient transfection of the cells was performed at a cell density of approximately $1.7 \times 10^{6}$ cells $\mathrm{mL}^{-1}$ culture volume using a total of $1 \mu \mathrm{g}$ of plasmid DNA and $2 \mu \mathrm{g}$ of linear 40-kDa polyethylenimine (Polysciences Inc., Germany) per mL culture volume. $48 \mathrm{~h}$ and $96 \mathrm{~h}$ after transfection, cells were supplemented with $0.5 \%$ (w/v) tryptone N1 (Organotechnie, France) and $0.25 \%(w / v) ~ D(+)$-glucose (Carl Roth, Germany). Soluble proteins were harvested after 120-144 h by centrifugation (10 $000 \mathrm{~g}, 15 \mathrm{~min}$, $\left.4^{\circ} \mathrm{C}\right)$.

\section{Purification of recombinantly expressed proteins}

After filtration through $0.45 \mu \mathrm{m}$ membrane filters (Merck Millipore, Germany), supernatants containing RBD or soluble Spike were concentrated and diafiltrated against $20 \mathrm{mM}$ sodium phosphate buffer containing $500 \mathrm{mM} \mathrm{NaCl}$ and $20 \mathrm{mM}$ imidazole (pH 7.4) using a Labscale TFF system equipped with a $5 \mathrm{kDa}$ cut-off Pellicon XL device (Merck Millipore). The His-tagged proteins were captured using a $5 \mathrm{~mL}$ HisTrap FF crude column connected to an ÄKTA pure 
chromatography system (both from Cytiva, United States). Bound proteins were eluted by applying a linear gradient of 20 to $500 \mathrm{mM}$ imidazole over 20 column volumes. ACE2-Fc variants were purified by affinity chromatography using a $5 \mathrm{~mL}$ HiTrap Protein A column (Cytiva) according to the manufacturer's instructions and $0.1 \mathrm{M}$ glycine- $\mathrm{HCl}$ ( $\mathrm{pH} 3.5$ ) for elution. Eluate fractions were immediately neutralized using $2 \mathrm{M}$ Tris ( $\mathrm{pH}$ 12.0). Fractions containing the protein of interest were pooled, concentrated using Vivaspin 20 Ultrafiltration Units (Sartorius, Germany) and dialyzed against PBS (pH 7.4) at $4^{\circ} \mathrm{C}$ overnight using SnakeSkin Dialysis Tubing (Thermo Fisher Scientific). The RBD was further purified by size exclusion chromatography (SEC) using a HiLoad 16/600 Superdex 200 pg column (Cytiva) eluted with PBS. All purified proteins were stored at $-80^{\circ} \mathrm{C}$ until further use.

\section{Enzymatic deglycosylation and desialylation of ACE2}

525 For deglycosylation of ACE2-wt-Fc and rshACE2, proteins $\left(2 \mathrm{mg} \mathrm{mL}^{-1}\right)$ were incubated with $180000 \mathrm{U} \mathrm{mL}^{-1}$ PNGase F (New England Biolabs, Unites States) in PBS (pH 7.4) for $24 \mathrm{~h}$ at $37^{\circ} \mathrm{C}$. Desialylation of ACE2-wt-Fc was performed with $2500 \mathrm{U} \mathrm{mL}^{-1}$ neuraminidase (New England Biolabs) in $50 \mathrm{mM}$ sodium citrate ( $\mathrm{pH}$ 5.0) under otherwise identical conditions. The deglycosylated or desialylated ACE2 variants were purified by preparative SEC using a HiLoad 530 16/600 Superdex 200 pg column eluted in PBS. The extent of enzymatic deglycosylation and desialylation was assessed by SDS-PAGE (Fig. S8), SEC-MALS (Fig. S9) and ESI-LC-MS/MS (Figs. S15 and S16).

\section{Bio-Layer Interferometry (BLI) measurements}

Interaction studies were performed on an Octet RED96e system using high precision streptavidin 535 (SAX) biosensors (both from ForteBio, United States). Thus, all capture molecules (ACE2-wtFc, ACE2-T92Q-Fc, ACE2-N322Q-Fc, ACE2-T92Q-N322Q-Fc, deglyco-ACE2-wt-Fc, desialoACE2-wt-Fc, rshACE2 and deglyco-rshACE2) were biotinylated using the EZ-Link Sulfo-NHSLC-Biotin kit (Thermo Fisher Scientific). Excess sulfo-NHS-LC-biotin was quenched by adding Tris- $\mathrm{HCl}$ buffer $(800 \mathrm{mM}, \mathrm{pH} 7.4)$ to a final concentration of $3 \mathrm{mM}$. Biotinylated proteins were further purified using PD-10 desalting columns (Cytiva) according to the manufacturer's protocol. All assays were conducted in PBS supplemented with $0.05 \%$ (v/v) Tween 20 and $0.1 \%$ (w/v) BSA (PBST-BSA) at $25^{\circ} \mathrm{C}$ with the plate shaking at $1000 \mathrm{rpm}$. The SAX biosensors were first equilibrated in PBST-BSA and then dipped into a $34 \mathrm{nM}$ solution of the respective 
biotinylated capture molecule until a signal threshold of $0.8 \mathrm{~nm}$ was reached. Subsequently, the biosensors were dipped into PBST-BSA for $90 \mathrm{sec}$ to record a baseline, before they were submerged into different concentrations of RBD or the Spike protein to record association rates. For binding analysis of trimeric Spike, all biosensors were dipped into 2-fold serial dilutions of the protein $(1.6-50 \mathrm{nM})$. To determine $K_{\mathrm{D}}$ values, titration of RBD was performed at different concentrations to cover a broad concentration range around the respective $K_{\mathrm{D}}$ value. ${ }^{64}$ Biosensors 555 biosensors was observed. Data were evaluated under consideration of the limit of detection (LOD) and limit of quantification (LOQ) as reported elsewhere. ${ }^{65,66}$ Each experiment was performed 3 times. Analysis was performed using the Octet data analysis software version 11.1.1.39 (ForteBio) according to the manufacturer's guidelines.

\section{SDS-PAGE}

SDS-PAGE was carried out using a 4-15\% MINI-PROTEAN TGX Stain-Free Protein Gel, the Mini-PROTEAN Tetra Vertical Electrophoresis Cell (both from Bio-Rad Laboratories Inc., United States) and SDS-PAGE running buffer (20 mM Tris, $200 \mathrm{mM}$ glycine, $0.1 \%$ (w/v) SDS). $1 \mu \mathrm{g}$ of each purified protein was mixed with SDS sample buffer (62.5 mM Tris/ $\mathrm{HCl}(\mathrm{pH} 6.8)$, $2.5 \%(\mathrm{w} / \mathrm{v}) \mathrm{SDS}, 10 \%(\mathrm{w} / \mathrm{v})$ glycerol, $0.01 \%(\mathrm{w} / \mathrm{v})$ bromophenol blue), heated to $70^{\circ} \mathrm{C}$ for 10 min and loaded onto the gel. For reducing conditions purified samples were mixed with SDSPAGE sample buffer containing $0.75 \mathrm{M} \beta$-mercaptoethanol and heated to $95^{\circ} \mathrm{C}$ for 5 min prior to loading. The PageRuler Unstained Protein Ladder (Thermo Fisher Scientific) was used as a size marker. Protein bands were visualized with the Gel Doc XR+ Imager (Bio-Rad Laboratories).

\section{Size-Exclusion Chromatography - Multi-Angle Light Scattering (SEC-MALS)}

Size-exclusion chromatography combined with multi-angle light scattering was performed to determine the homogeneity and the native molecular mass of all proteins under study. Analyses were performed on an LC20 Prominence HPLC equipped with a refractive index detector RID- 
10A and the photodiode array detector SPD-M20A (all from Shimadzu, Japan). In-line MALS was analyzed either with a miniDAWN TREOS II MALS (for analysis of Spike) or a Heleos Dawn8+ plus QELS apparatus (Wyatt Technology, United States). Prior to analysis, all proteins were centrifuged $\left(16000 \mathrm{~g}, 10 \mathrm{~min}, 20^{\circ} \mathrm{C}\right)$ and filtered $(0.1 \mu \mathrm{m}$ Ultrafree-MC filter, Merck Millipore). Proper performance of the MALS detectors was validated with bovine serum albumin. Purified Spike was analyzed by injection of a total of $50 \mu \mathrm{g}$ onto a Superose 6 Increase 10/300 GL column (Cytiva) at a flow rate of $0.25 \mathrm{~mL} \mathrm{~min}^{-1}$. The mobile-phase buffer used was PBS supplemented with $10 \%$ glycerol ( $\mathrm{pH} 7.4$ ). All other proteins were analyzed by using a Superdex 200 10/300 GL column (Cytiva) equilibrated with PBS plus $200 \mathrm{mM} \mathrm{NaCl}$ (pH 7.4). A total of $25 \mu \mathrm{g}$ of each protein was injected and experiments were performed at a flow rate of 0.75 $\mathrm{mL} \mathrm{min}^{-1}$. Data were analyzed using the ASTRA 6 software (Wyatt Technology).

\section{Differential scanning calorimetry (DSC)}

DSC experiments were performed using a MicroCal PEAQ-DSC Automated system (Malvern Panalytical, Germany), using $2.5 \mu \mathrm{M}$ protein solutions in PBS ( $\mathrm{pH} 7.4$ ). The heating was performed from $20^{\circ} \mathrm{C}$ to $100^{\circ} \mathrm{C}$ at a rate of $1^{\circ} \mathrm{C} / \mathrm{min}$. The protein solution was then cooled in situ and an identical thermal scan was run to obtain the baseline for subtraction from the first scan.

590 All measurements were performed in triplicates. Fitting was done with Origin 7.0 for DSC software using the non-2-state transition model.

\section{Peptide mapping and glycopeptide analysis}

All samples were analysed as in-solution proteolytic digests of the respective proteins by LCESI-MS/MS. For this, the $\mathrm{pH}$ of the samples was first adjusted to $\mathrm{pH} 7.8$ by the addition of $1 \mathrm{M}$ HEPES ( $\mathrm{pH} 7.8$ ) to a final concentration of $100 \mathrm{mM}$. The samples were then chemically reduced and S-alkylated, using a final concentration of $10 \mathrm{mM}$ dithiothreitol for $30 \mathrm{~min}$ at $56^{\circ} \mathrm{C}$, and a final concentration of $20 \mathrm{mM}$ iodoacetamide for $30 \mathrm{~min}$ at room temperature in the dark. To maximize sequence coverage, proteins were digested for $18 \mathrm{~h}$ at $37^{\circ} \mathrm{C}$ with chymotrypsin (Roche, Germany), followed by $3 \mathrm{~h}$ at $37^{\circ} \mathrm{C}$ using trypsin (Promega). All proteolytic digests were acidified to $\mathrm{pH} 2$ by addition of $10 \%$ formic acid and directly analyzed by LC-ESI-MS/MS, using a capillary BioBasic C18 reversed-phase column (BioBasic-18, $150 \times 0.32 \mathrm{~mm}, 5 \mu \mathrm{m}$, Thermo Fisher Scientific), installed in a Dionex Ultimate U3000 HPLC system (Thermo Fisher Scientific), developing a linear gradient from 95\% eluent A (80 mM ammonium formate, $\mathrm{pH} 3.0$, 
in HPLC-grade water) to $65 \%$ eluent B (80\% acetonitrile in $80 \mathrm{mM}$ ammonium formate, $\mathrm{pH} 3.0)$ 610 selected for MS/MS fragmentation. Instrument calibration was performed using a commercial ESI calibration mixture (Agilent Technologies). Site-specific profiling of protein glycosylation was performed using the dedicated Q-TOF data-analysis software packages Data Analyst (Bruker Daltonics) and Protein Scape (Bruker Daltonics), in conjunction with the MS/MS search engine MASCOT (Matrix Sciences Inc., United States) for automated peptide identification.

\section{ACE2 activity assays}

Enzymatic activity of ACE2 was determined and quantified as described previously, ${ }^{37}$ using 100 $\mu \mathrm{M}$ 7-methoxycoumarin-4-yl-acetyl-Ala-Pro-Lys-2,4-dinitrophenyl (Bachem, Switzerland) as substrate.

\section{SARS-CoV-2 neutralization assays}

620 All work with infectious SARS-CoV-2 was performed under BSL-3 conditions. Vero E6 cells (Biomedica, Austria) were grown in Minimum Essential Medium (MEM) containing Earle's Salts, $1 \%$ penicillin/streptomycin stock solution and $2 \mathrm{mM} \mathrm{L-glutamine} \mathrm{(all} \mathrm{from} \mathrm{Thermo} \mathrm{Fisher}$ Scientific), supplemented with $5 \%$ fetal bovine serum (FBS), at $37^{\circ} \mathrm{C}$ and $5 \% \mathrm{CO}_{2}$. A German 2019-nCoV isolate (Ref-SKU: 026V-03883, Charité, Berlin, Germany) was propagated in Vero E6 cells. The TCID50 titer of virus stocks was determined by the Reed-Munch method ${ }^{67}$ and converted to plaque-forming units (pfu) using the conversion factor 0.7 (https://www.atcc.org/support/technical-support/faqs/converting-tcid-50-to-plaque-formingunits-pfu). Vero E6 cells were seeded in 48 -well cell culture plates $\left(3 \times 10^{4}\right.$ cells per well) in MEM supplemented with $2 \%$ FBS overnight to reach approximately $80 \%$ confluence on the day of infection. ACE2 variants (final concentrations: 10-100 $\mu \mathrm{g} / \mathrm{mL}$ ) were preincubated with $60 \mathrm{pfu}$ SARS-CoV-2 for $30 \mathrm{~min}$ at $37^{\circ} \mathrm{C}$ under constant shaking (300 rpm). After preincubation, Vero E6 cells were infected for $1 \mathrm{~h}$ at $37^{\circ} \mathrm{C}$ with samples containing either SARS-CoV-2 and ACE2 variants or solely SARS-CoV-2 (untreated controls) at a multiplicity of infection (MOI) of 0.002. 
Subsequently, cells were washed two times with MEM to remove unadsorbed virus. After incubation for $24 \mathrm{~h}$ at $37^{\circ} \mathrm{C}$ in MEM supplemented with $2 \% \mathrm{FBS}$, viral RNA was extracted from the culture supernatant using the QiaAmp Viral RNA Minikit (Qiagen, Germany), according to the manufacturer's protocol. SARS-CoV-2 replication was quantified via RT-qPCR using the QuantiTect Multiplex RT-qPCR Kit (Qiagen) with a Rotor Gene Q cycler (Qiagen). The reactions were performed in a total volume of $25 \mu \mathrm{L}$ at $50^{\circ} \mathrm{C}$ for $30 \mathrm{~min}$ followed by $95^{\circ} \mathrm{C}$ for 15 min and 45 cycles of $95^{\circ} \mathrm{C}$ for $3 \mathrm{~s}$ and $55^{\circ} \mathrm{C}$ for $30 \mathrm{~s}$. Forward primer: 2019-nCoV_N1-F 5'GACCCCAAAATCAGCGAAAT-3'; reverse primer: 2019-nCoV_N1-R 5'TCTGGTTACTGCCAGTTGAATCTG-3'; probe: 2019-nCoV_N1-P 5'-FAMACCCCGCATTACGTTTGGTGGACC-BHQ1-3'. Statistical analyses were conducted using GraphPad Prism 8. Significance was determined by Kruskal-Wallis, comparing the mean rank of 645 the ACE2-wt-Fc group with the mean rank of every other group $(*, \mathrm{P}<0.05 ; * *, \mathrm{P}<0.01 ; * * *$, $\mathrm{P}<0.001)$.

SARS-CoV-2 neutralization assays were also performed independently in another laboratory using a different virus isolate. For these assays, Vero E6 cells (ATCC, United States) were grown in Dulbecco's Modified Eagle's Medium (DMEM, Thermo Fisher Scientific) 650 supplemented with $1 \%$ non-essential amino acid stock solution (Thermo Fisher Scientific), 10 $\mathrm{mM}$ HEPES (Thermo Fisher Scientific) and $10 \% \mathrm{FBS}$ at $37^{\circ} \mathrm{C}$ and $5 \% \mathrm{CO}_{2}$. SARS-CoV-2 isolated from a nasopharyngeal sample of a Swedish COVID-19 patient (GenBank accession number MT093571) was propagated in Vero E6 cells. Virus was titered using a plaque assay as previously described ${ }^{68}$ with fixation of cells $72 \mathrm{~h}$ post infection. Vero E6 cells were treated and 655 infected as described previously. ${ }^{5}$ Briefly, Vero E6 cells were seeded in 48 -well plates $\left(5 \times 10^{4}\right.$ cells per well) in DMEM containing 10\% FBS. $24 \mathrm{~h}$ post-seeding, ACE2 variants (final concentrations: $50-200 \mu \mathrm{g} / \mathrm{mL}$ ) were mixed with $10^{6}$ pfu SARS-CoV-2 (MOI: 20) in a final volume of $100 \mu \mathrm{l}$ DMEM containing $5 \% \mathrm{FBS}$, incubated for $30 \mathrm{~min}$ at $37^{\circ} \mathrm{C}$ and then added to the cells. $15 \mathrm{~h}$ post-infection, cells were washed 3 times with PBS and then lysed using Trizol 660 (Thermo Fisher Scientific) before analysis by RT-qPCR to quantify the content of SARS-CoV-2 RNA as described. ${ }^{5}$

\section{Acknowledgments}


We thank Florian Krammer (Icahn School of Medicine at Mount Sinai, NY, United States) for providing the constructs used for production of recombinant Spike and RBD. pcDNA3sACE2(WT)-Fc(IgG1) and pcDNA3-sACE2-T92Q-Fc(IgG1) were used with the kind permission of Erik Procko (University of Illinois, IL, United States). Transfection-grade pCAGGS plasmids were provided by Rainer Hahn and Gerald Striedner (University of Natural 670 Resources and Life Sciences Vienna, Austria) in the framework of the BOKU COVID-19 Initiative. The authors thank Irene Schaffner and Jakob Wallner (BOKU Core Facility Biomolecular \& Cellular Analysis) for assisting in BLI measurements, Gerhard Stadlmayr (University of Natural Resources and Life Sciences Vienna, Austria) for performing SEC-MALS analysis of Spike and ForteBio for supporting the BOKU COVID-19 Initiative with SAX 675 biosensors. The high-performance computing center (HLRS) of the University of Stuttgart is gratefully acknowledged for providing computational resources.

\section{Funding}

This project is supported by the $\mathrm{PhD}$ programme BioToP funded by the Austrian Science Fund (grant No. W1224-B09), Vienna Science and Technology Fund (WWTF; grant No. COV20015), and funding from the Innovative Medicines Initiative 2 Joint Undertaking (JU; grant agreement No. 101005026). The JU receives support from the European Union's Horizon 2020 research and innovation programme and EFPIA. J.W.P. is a recipient of a DOC Fellowship of the Austrian Academy of Sciences (ÖAW) at the Institute for Molecular Modeling and 685 Simulation at the University of Natural Resources and Life Sciences, Vienna (Grant No. 24987). J.M.P. and the research leading to these results has received funding from the T. von Zastrow foundation, the FWF Wittgenstein award (grant No. Z271-B19), the Austrian Academy of Sciences, the Canada 150 Research Chairs Program in Functional Genetics (grant No. F180133), and the Canadian Institutes of Health Research COVID-19 grants F20-02343 and F2002015.

\section{Author contributions}

Tümay Capraz: investigation, formal analysis, writing original draft, visualization; Esther Föderl-Höbenreich: investigation, formal analysis, visualization, writing review and editing; 
695 Clemens Grünwald-Gruber: investigation; Nikolaus F. Kienzl: investigation, formal analysis, visualization, writing review and editing; Elisabeth Laurent: investigation, formal analysis, writing original draft, visualization; Lukas Mach: investigation, supervision, formal analysis, writing original draft, writing review and editing; Daniel Maresch: investigation; Ali Mirazimi: supervision; Vanessa Monteil: investigation; Janine Niederhöfer: investigation; Chris Oostenbrink: conceptualization, supervision, writing original draft, writing review and editing; Josef M. Penninger: initiation of the project, conceptualization, writing review and editing; Jan W. Perthold: investigation, supervision, writing original draft; Johannes Stadlmann: conceptualization, investigation, supervision, formal analysis, writing original draft, writing review and editing; Gerald Wirnsberger: investigation, supervision, resources; Kurt Zatloukal: supervision, resources, writing review and editing.

\section{Competing interests}

J.M.P. declares a conflict of interest as a founder, supervisory board member, and shareholder of Apeiron Biologics. J.N. and G.W. are employees of Apeiron Biologics. Apeiron holds a patent

on the use of ACE2 for the treatment of lung, heart, or kidney injury and applied for a patent to treat COVID-19 with rshACE2.

\section{Data and materials availability}

Molecular models and simulation trajectories are available through the BioExcel COVID-19 Molecular Structure and Therapeutics Hub (https://covid.bioexcel.eu/simulations/). 


\section{References}

720 1. Zhu, N., Zhang, D., Wang, W., Li, X., Yang, B., Song, J., Zhao, X., Huang, B., Shi, W., Lu, R., Niu, P., Zhan, F., Ma, X., Wang, D., Xu, W., Wu, G., Gao, G. F., Tan, W., China Novel Coronavirus, I., et al. (2020) A Novel Coronavirus from Patients with Pneumonia in China, 2019. N Engl J Med 382, 727-733. doi: 10.1056/NEJMoa2001017

2. Wu, F., Zhao, S., Yu, B., Chen, Y. M., Wang, W., Song, Z. G., Hu, Y., Tao, Z. W., Tian, J. H., Pei, Y. Y., Yuan, M. L., Zhang, Y. L., Dai, F. H., Liu, Y., Wang, Q. M., Zheng, J. J., Xu, L., Holmes, E. C., and Zhang, Y. Z. (2020) A new coronavirus associated with human respiratory disease in China. Nature 579, 265-269. doi: 10.1038/s41586-0202008-3

3. Zhou, P., Yang, X. L., Wang, X. G., Hu, B., Zhang, L., Zhang, W., Si, H. R., Zhu, Y., Li, B., Huang, C. L., Chen, H. D., Chen, J., Luo, Y., Guo, H., Jiang, R. D., Liu, M. Q., Chen, Y., Shen, X. R., Wang, X., et al. (2020) A pneumonia outbreak associated with a new coronavirus of probable bat origin. Nature 579, 270-273. doi: 10.1038/s41586-020-20127

4. Wang, Q., Zhang, Y., Wu, L., Niu, S., Song, C., Zhang, Z., Lu, G., Qiao, C., Hu, Y., Yuen, K. Y., Wang, Q., Zhou, H., Yan, J., and Qi, J. (2020) Structural and Functional Basis of SARS-CoV-2 Entry by Using Human ACE2. Cell 181, 894-904 e899. doi: 10.1016/j.cell.2020.03.045

5. Monteil, V., Kwon, H., Prado, P., Hagelkruys, A., Wimmer, R. A., Stahl, M., Leopoldi, A., Garreta, E., Hurtado Del Pozo, C., Prosper, F., Romero, J. P., Wirnsberger, G.,

6. Monteil, V., Dyczynski, M., Lauschke, V. M., Kwon, H., Wirnsberger, G., Youhanna, S., Zhang, H., Slutsky, A. S., Conder, R., Montserrat, N., Mirazimi, A., and Penninger, J. M. (2020) Inhibition of SARS-CoV-2 Infections in Engineered Human Tissues Using Clinical-Grade Soluble Human ACE2. Cell 181, 905-913 e907. doi: 10.1016/j.cell.2020.04.004 Zhang, H., Slutsky, A. S., Hurtado Del Pozo, C., Horn, M., Montserrat, N., Penninger, J. M., and Mirazimi, A. (2021) Human soluble ACE2 improves the effect of remdesivir in SARS-CoV-2 infection. EMBO Mol Med 13, e13426. doi: 10.15252/emmm. 202013426

7. Zoufaly, A., Poglitsch, M., Aberle, J. H., Hoepler, W., Seitz, T., Traugott, M., Grieb, A., Pawelka, E., Laferl, H., Wenisch, C., Neuhold, S., Haider, D., Stiasny, K., Bergthaler, A., Puchhammer-Stoeckl, E., Mirazimi, A., Montserrat, N., Zhang, H., Slutsky, A. S., et al. (2020) Human recombinant soluble ACE2 in severe COVID-19. Lancet Respir Med 8, 1154-1158. doi: 10.1016/S2213-2600(20)30418-5

8. Barnes, C. O., Jette, C. A., Abernathy, M. E., Dam, K. A., Esswein, S. R., Gristick, H. B., Malyutin, A. G., Sharaf, N. G., Huey-Tubman, K. E., Lee, Y. E., Robbiani, D. F., Nussenzweig, M. C., West, A. P., Jr., and Bjorkman, P. J. (2020) SARS-CoV-2 neutralizing antibody structures inform therapeutic strategies. Nature 588, 682-687. doi: 10.1038/s41586-020-2852-1

9. Chan, J. F. W., Oh, Y. J., Yuan, S., Chu, H., Yeung, M. L., Canena, D., Chan, C. C. S., Poon, V. K. M., Chan, C. C. Y., Zhang, A. J., Cai, J. P., Wen, L., Chik, K. K. H., Shuai, H., Wang, Y., Hou, Y., Luo, C., Chan, W. M., Sit, K. Y., et al. (2021) A molecularly 
engineered, broad-spectrum anti-coronavirus lectin inhibits SARS-CoV-2 and MERSCoV infection in vivo. Submitted to Nature. doi:

10. Hoffmann, D., Mereiter, S., Jin Oh, Y., Monteil, V., Elder, E., Zhu, R., Canena, D., Hain, L., Laurent, E., Grunwald-Gruber, C., Klausberger, M., Jonsson, G., Kellner, M. J., Novatchkova, M., Ticevic, M., Chabloz, A., Wirnsberger, G., Hagelkruys, A., Altmann, F., et al. (2021) Identification of lectin receptors for conserved SARS-CoV-2 glycosylation sites. EMBO J 10.15252/embj.2021108375, e108375. doi: 10.15252/embj.2021108375

11. Weisblum, Y., Schmidt, F., Zhang, F., DaSilva, J., Poston, D., Lorenzi, J. C., Muecksch, F., Rutkowska, M., Hoffmann, H. H., Michailidis, E., Gaebler, C., Agudelo, M., Cho, A., Wang, Z., Gazumyan, A., Cipolla, M., Luchsinger, L., Hillyer, C. D., Caskey, M., et al. (2020) Escape from neutralizing antibodies by SARS-CoV-2 spike protein variants. Elife 9, e61312. doi: 10.7554/eLife.61312

12. Li, D., Sempowski, G. D., Saunders, K. O., Acharya, P., and Haynes, B. F. (2021) SARSCoV-2 Neutralizing Antibodies for COVID-19 Prevention and Treatment. Annu Rev Med 10.1146/annurev-med-042420-113838. doi: 10.1146/annurev-med-042420-113838

13. Yuan, M., Huang, D., Lee, C. D., Wu, N. C., Jackson, A. M., Zhu, X., Liu, H., Peng, L., van Gils, M. J., Sanders, R. W., Burton, D. R., Reincke, S. M., Pruss, H., Kreye, J., Nemazee, D., Ward, A. B., and Wilson, I. A. (2021) Structural and functional ramifications of antigenic drift in recent SARS-CoV-2 variants. Science 373, 818-823. doi: 10.1126/science.abh1139

14. Glasgow, A., Glasgow, J., Limonta, D., Solomon, P., Lui, I., Zhang, Y., Nix, M. A., Rettko, N. J., Zha, S., Yamin, R., Kao, K., Rosenberg, O. S., Ravetch, J. V., Wiita, A. P., Leung, K. K., Lim, S. A., Zhou, X. X., Hobman, T. C., Kortemme, T., et al. (2020) Engineered ACE2 receptor traps potently neutralize SARS-CoV-2. Proc Natl Acad Sci U $S$ A 117, 28046-28055. doi: 10.1073/pnas.2016093117

15. Chan, K. K., Dorosky, D., Sharma, P., Abbasi, S. A., Dye, J. M., Kranz, D. M., Herbert, A. S., and Procko, E. (2020) Engineering human ACE2 to optimize binding to the spike protein of SARS coronavirus 2. Science 369, 1261-1265. doi: 10.1126/science.abc0870

16. Lan, J., Ge, J., Yu, J., Shan, S., Zhou, H., Fan, S., Zhang, Q., Shi, X., Wang, Q., Zhang, L., and Wang, X. (2020) Structure of the SARS-CoV-2 spike receptor-binding domain bound to the ACE2 receptor. Nature 581, 215-220. doi: 10.1038/s41586-020-2180-5

17. Yan, R., Zhang, Y., Li, Y., Xia, L., Guo, Y., and Zhou, Q. (2020) Structural basis for the recognition of SARS-CoV-2 by full-length human ACE2. Science 367, 1444-1448. doi: 10.1126/science.abb2762

18. Walls, A. C., Park, Y. J., Tortorici, M. A., Wall, A., McGuire, A. T., and Veesler, D. (2020) Structure, Function, and Antigenicity of the SARS-CoV-2 Spike Glycoprotein. Cell 181, 281-292 e286. doi: 10.1016/j.cell.2020.02.058

19. Wrapp, D., Wang, N., Corbett, K. S., Goldsmith, J. A., Hsieh, C. L., Abiona, O., Graham, B. S., and McLellan, J. S. (2020) Cryo-EM structure of the 2019-nCoV spike in the prefusion conformation. Science 367, 1260-1263. doi: 10.1126/science.abb2507

20. Watanabe, Y., Allen, J. D., Wrapp, D., McLellan, J. S., and Crispin, M. (2020) Sitespecific glycan analysis of the SARS-CoV-2 spike. Science 369, 330-333. doi: 10.1126/science.abb9983

805 21. Zhao, P., Praissman, J. L., Grant, O. C., Cai, Y., Xiao, T., Rosenbalm, K. E., Aoki, K., Kellman, B. P., Bridger, R., Barouch, D. H., Brindley, M. A., Lewis, N. E., Tiemeyer, 
M., Chen, B., Woods, R. J., and Wells, L. (2020) Virus-Receptor Interactions of Glycosylated SARS-CoV-2 Spike and Human ACE2 Receptor. Cell Host Microbe 28, 586-601 e586. doi: 10.1016/j.chom.2020.08.004

22. Casalino, L., Gaieb, Z., Goldsmith, J. A., Hjorth, C. K., Dommer, A. C., Harbison, A. M., Fogarty, C. A., Barros, E. P., Taylor, B. C., McLellan, J. S., Fadda, E., and Amaro, R. E. (2020) Beyond Shielding: The Roles of Glycans in the SARS-CoV-2 Spike Protein. ACS Cent Sci 6, 1722-1734. doi: 10.1021/acscentsci.0c01056

23. Sikora, M., von Bulow, S., Blanc, F. E. C., Gecht, M., Covino, R., and Hummer, G. (2021) Computational epitope map of SARS-CoV-2 spike protein. PLoS Comput Biol 17, e1008790. doi: 10.1371/journal.pcbi.1008790

24. Zimmerman, M. I., Porter, J. R., Ward, M. D., Singh, S., Vithani, N., Meller, A., Mallimadugula, U. L., Kuhn, C. E., Borowsky, J. H., Wiewiora, R. P., Hurley, M. F. D., Harbison, A. M., Fogarty, C. A., Coffland, J. E., Fadda, E., Voelz, V. A., Chodera, J. D., and Bowman, G. R. (2021) SARS-CoV-2 simulations go exascale to predict dramatic spike opening and cryptic pockets across the proteome. Nat Chem 13, 651-659. doi: 10.1038/s41557-021-00707-0

25. Mehdipour, A. R., and Hummer, G. (2021) Dual nature of human ACE2 glycosylation in binding to SARS-CoV-2 spike. Proc Natl Acad Sci $U$ S A 118. doi: 10.1073/pnas.2100425118

26. Sztain, T., Ahn, S. H., Bogetti, A. T., Casalino, L., Goldsmith, J. A., Seitz, E., McCool, R. S., Kearns, F. L., Acosta-Reyes, F., Maji, S., Mashayekhi, G., McCammon, J. A., Ourmazd, A., Frank, J., McLellan, J. S., Chong, L. T., and Amaro, R. E. (2021) A glycan gate controls opening of the SARS-CoV-2 spike protein. Nat Chem 10.1038/s41557-02100758-3. doi: 10.1038/s41557-021-00758-3

27. Cong, Y., Feng, Y., Ni, H., Zhi, F., Miao, Y., Fang, B., Zhang, L., and Zhang, J. Z. H. (2021) Anchor-Locker Binding Mechanism of the Coronavirus Spike Protein to Human ACE2: Insights from Computational Analysis. Journal of Chemical Information and Modeling 61, 3529-3542. doi: 10.1021/acs.jcim.1c00241

28. Delgado, J. M., Duro, N., Rogers, D. M., Tkatchenko, A., Pandit, S. A., and Varma, S. (2021) Molecular basis for higher affinity of SARS-CoV-2 spike RBD for human ACE2 receptor. Proteins 89, 1134-1144. doi: 10.1002/prot.26086

29. Yang, Q., Hughes, T. A., Kelkar, A., Yu, X., Cheng, K., Park, S., Huang, W. C., Lovell, J. F., and Neelamegham, S. (2020) Inhibition of SARS-CoV-2 viral entry upon blocking $\mathrm{N}$ - and O-glycan elaboration. Elife 9. doi: 10.7554/eLife.61552

30. Allen, J. D., Watanabe, Y., Chawla, H., Newby, M. L., and Crispin, M. (2021) Subtle Influence of ACE2 Glycan Processing on SARS-CoV-2 Recognition. J Mol Biol 433, 166762. doi: 10.1016/j.jmb.2020.166762

31. Sun, Z., Ren, K., Zhang, X., Chen, J., Jiang, Z., Jiang, J., Ji, F., Ouyang, X., and Li, L. (2020) Mass Spectrometry Analysis of Newly Emerging Coronavirus HCoV-19 Spike Protein and Human ACE2 Reveals Camouflaging Glycans and Unique Post-Translational Modifications. Engineering (Beijing) 10.1016/j.eng.2020.07.014. doi: 10.1016/j.eng.2020.07.014

32. Grant, O. C., Montgomery, D., Ito, K., and Woods, R. J. (2020) Analysis of the SARSCoV-2 spike protein glycan shield reveals implications for immune recognition. Sci Rep 10, 14991. doi: 10.1038/s41598-020-71748-7 
33. Li, Q., Wu, J., Nie, J., Zhang, L., Hao, H., Liu, S., Zhao, C., Zhang, Q., Liu, H., Nie, L., Qin, H., Wang, M., Lu, Q., Li, X., Sun, Q., Liu, J., Zhang, L., Li, X., Huang, W., et al. (2020) The Impact of Mutations in SARS-CoV-2 Spike on Viral Infectivity and Antigenicity. Cell 182, 1284-1294 e1289. doi: 10.1016/j.cell.2020.07.012

34. Mori, T., Jung, J., Kobayashi, C., Dokainish, H. M., Re, S., and Sugita, Y. (2021) Elucidation of interactions regulating conformational stability and dynamics of SARSCoV-2 S-protein. Biophys J 120, 1060-1071. doi: 10.1016/j.bpj.2021.01.012

35. Barros, E. P., Casalino, L., Gaieb, Z., Dommer, A. C., Wang, Y., Fallon, L., Raguette, L., Belfon, K., Simmerling, C., and Amaro, R. E. (2021) The flexibility of ACE2 in the context of SARS-CoV-2 infection. Biophys $J$ 120, 1072-1084. doi: 10.1016/j.bpj.2020.10.036

36. Lobner, E., Humm, A. S., Goritzer, K., Mlynek, G., Puchinger, M. G., Hasenhindl, C., Ruker, F., Traxlmayr, M. W., Djinovic-Carugo, K., and Obinger, C. (2017) Fcab-HER2 Interaction: a Menage a Trois. Lessons from X-Ray and Solution Studies. Structure 25, 878-889 e875. doi: 10.1016/j.str.2017.04.014

37. Vickers, C., Hales, P., Kaushik, V., Dick, L., Gavin, J., Tang, J., Godbout, K., Parsons, T., Baronas, E., Hsieh, F., Acton, S., Patane, M., Nichols, A., and Tummino, P. (2002) Hydrolysis of biological peptides by human angiotensin-converting enzyme-related carboxypeptidase. J Biol Chem 277, 14838-14843. doi: 10.1074/jbc.M200581200

38. Chu, H., Hu, B., Huang, X., Chai, Y., Zhou, D., Wang, Y., Shuai, H., Yang, D., Hou, Y., Zhang, X., Yuen, T. T., Cai, J. P., Zhang, A. J., Zhou, J., Yuan, S., To, K. K., Chan, I. H., Sit, K. Y., Foo, D. C., et al. (2021) Host and viral determinants for efficient SARS-CoV-2 infection of the human lung. Nat Commun 12, 134. doi: 10.1038/s41467-020-20457-w

39. $\mathrm{Xu}, \mathrm{C}$., and $\mathrm{Ng}, \mathrm{D}$. T. (2015) Glycosylation-directed quality control of protein folding. Nat Rev Mol Cell Biol 16, 742-752. doi: 10.1038/nrm4073

40. Waterhouse, A., Bertoni, M., Bienert, S., Studer, G., Tauriello, G., Gumienny, R., Heer, F. T., de Beer, T. A. P., Rempfer, C., Bordoli, L., Lepore, R., and Schwede, T. (2018) SWISS-MODEL: homology modelling of protein structures and complexes. Nucleic Acids Res 46, W296-W303. doi: 10.1093/nar/gky427

41. Turupcu, A., and Oostenbrink, C. (2017) Modeling of Oligosaccharides within Glycoproteins from Free-Energy Landscapes. Journal of Chemical Information and Modeling 57, 2222-2236. doi: 10.1021/acs.jcim.7b00351

42. Huber, T., Torda, A. E., and van Gunsteren, W. F. (1994) Local elevation: A method for improving the searching properties of molecular dynamics simulations. Journal of Computer-Aided Molecular Design 8, 695 - 708. doi: 10.1007/Bf00124016

43. Peric-Hassler, L., Hansen, H. S., Baron, R., and Hünenberger, P. H. (2010) Conformational properties of glycose-based disaccharides investigated using molecular dynamics simulations with local elevation umbrella sampling. Carbohydrate research 345, 1781 - 1801. doi: 10.1016/j.carres.2010.05.026

44. Eichenberger, A. P., Allison, J. R., Dolenc, J., Geerke, D. P., Horta, B. A. C., Meier, K., Oostenbrink, C., Schmid, N., Steiner, D., Wang, D. Q., and van Gunsteren, W. F. (2011) GROMOS plus plus Software for the Analysis of Biomolecular Simulation Trajectories. Journal of Chemical Theory and Computation 7, 3379-3390. doi: 10.1021/Ct2003622

45. Chys, P., and Chacon, P. (2013) Random Coordinate Descent with Spinor-matrices and Geometric Filters for Efficient Loop Closure. Journal of Chemical Theory and Computation 9, 1821-1829. doi: 10.1021/ct300977f 
46. Lopez-Blanco, J. R., Canosa-Valls, A. J., Li, Y., and Chacon, P. (2016) RCD+: Fast loop modeling server. Nucleic Acids Res 44, W395-400. doi: 10.1093/nar/gkw395

900 47. Reif, M. M., Hunenberger, P. H., and Oostenbrink, C. (2012) New Interaction Parameters for Charged Amino Acid Side Chains in the GROMOS Force Field. Journal of Chemical Theory and Computation 8, 3705-3723. doi: 10.1021/Ct300156h

48. Reif, M. M., Winger, M., and Oostenbrink, C. (2013) Testing of the GROMOS ForceField Parameter Set 54A8: Structural Properties of Electrolyte Solutions, Lipid Bilayers, and Proteins. Journal of Chemical Theory and Computation 9, 1247-1264. doi: 10.1021/Ct300874c

49. Pol-Fachin, L., Rusu, V. H., Verli, H., and Lins, R. D. (2012) GROMOS 53A6GLYC, an Improved GROMOS Force Field for Hexopyranose-Based Carbohydrates. Journal of Chemical Theory and Computation 8, 4681-4690. doi: 10.1021/ct300479h

910 50. Pol-Fachin, L., Verli, H., and Lins, R. D. (2014) Extension and validation of the GROMOS 53A6(GLYC) parameter set for glycoproteins. J Comput Chem 35, 20872095. doi: $10.1002 /$ jcc. 23721

51. Schmid, N., Christ, C. D., Christen, M., Eichenberger, A. P., and van Gunsteren, W. F. (2012) Architecture, implementation and parallelisation of the GROMOS software for biomolecular simulation. Computer Physics Communications 183, 890 - 903. doi: 10.1016/j.cpc.2011.12.014

52. Berendsen, H. J. C., Postma, J. P. M., van Gunsteren, W. F., and Hermans, J. (1981) Interaction models for water in relation to protein hydration, In Intermolecular Forces (Pullman, B., Ed.), pp 331-342, Reidel, Dordrecht, The Netherlands. doi:

920 53. Hockney, R. W. (1970) The potential calculations and some applications. Methods in Computational Physics 9, 136 - 211. doi:

54. Hockney, R. W., and Eastwood, B. J. (1988) Computer simulation using particles 10.1201/9780367806934, Institute of Physics Publishing, Bristol. doi: $10.1201 / 9780367806934$

925 55. Hess, B., Bekker, H., Berendsen, H. J. C., and Fraaije, J. G. E. M. (1997) LINCS: A linear constraint solver for molecular simulations. Journal of Computational Chemistry $18,1463 \quad$ - 1472. doi: 10.1002/(SICI)1096-987X(199709)18:12<1463::AIDJCC4>3.0.CO;2-H

56. Berendsen, H. J. C., Postma, J. P. M., van Gunsteren, W. F., DiNola, A., and Haak, J. R. (1984) Molecular-dynamics with coupling to an external bath. Journal of Chemical Physics 81, 3684-3690. doi:

57. Bussi, G., Donadio, D., and Parrinello, M. (2007) Canonical sampling through velocity rescaling. J Chem Phys 126, 014101. doi: 10.1063/1.2408420

58. Parinello, M., and Rahman, A. (1981) Polymorphic transitions in single crystals: A new molecular dynamic method. J. Applied Physics. 52, 7182 - 7190. doi: 10.1063/1.328693

935

59. Nosé, S., and Klein, M. L. (1983) Constant pressure molecular dynamics for molecular systems. Molecular Physics 50, 1055 - 1076. doi: 10.1080/00268978300102851

60. Briones, R., Blau, C., Kutzner, C., de Groot, B. L., and Aponte-Santamaria, C. (2019) GROmarhos: A GROMACS-Based Toolset to Analyze Density Maps Derived from Molecular Dynamics Simulations. Biophys J 116, 4-11. doi: 10.1016/j.bpj.2018.11.3126

61. Durocher, Y., Perret, S., and Kamen, A. (2002) High-level and high-throughput recombinant protein production by transient transfection of suspension-growing human 293-EBNA1 cells. Nucleic Acids Res 30, E9. doi: 10.1093/nar/30.2.e9 
62. Amanat, F., Stadlbauer, D., Strohmeier, S., Nguyen, T. H. O., Chromikova, V., McMahon, M., Jiang, K., Arunkumar, G. A., Jurczyszak, D., Polanco, J., BermudezGonzalez, M., Kleiner, G., Aydillo, T., Miorin, L., Fierer, D. S., Lugo, L. A., Kojic, E. M., Stoever, J., Liu, S. T. H., et al. (2020) A serological assay to detect SARS-CoV-2 seroconversion in humans. Nat Med 26, 1033-1036. doi: 10.1038/s41591-020-0913-5

63. Stadlbauer, D., Amanat, F., Chromikova, V., Jiang, K., Strohmeier, S., Arunkumar, G. A., Tan, J., Bhavsar, D., Capuano, C., Kirkpatrick, E., Meade, P., Brito, R. N., Teo, C., McMahon, M., Simon, V., and Krammer, F. (2020) SARS-CoV-2 Seroconversion in Humans: A Detailed Protocol for a Serological Assay, Antigen Production, and Test Setup. Curr Protoc Microbiol 57, e100. doi: 10.1002/cpmc.100

64. Hulme, E. C., and Trevethick, M. A. (2010) Ligand binding assays at equilibrium: validation and interpretation. Br J Pharmacol 161, 1219-1237. doi: 10.1111/j.14765381.2009.00604.x

65. Armbruster, D. A., and Pry, T. (2008) Limit of blank, limit of detection and limit of quantitation. Clin Biochem Rev 29 Suppl 1, S49-52. doi:

66. Carvalho, S. B., Moreira, A. S., Gomes, J., Carrondo, M. J. T., Thornton, D. J., Alves, P. M., Costa, J., and Peixoto, C. (2018) A detection and quantification label-free tool to speed up downstream processing of model mucins. PLoS One 13, e0190974. doi: 10.1371/journal.pone.0190974

67. Ramakrishnan, M. A. (2016) Determination of $50 \%$ endpoint titer using a simple formula. World J Virol 5, 85-86. doi: 10.5501/wjv.v5.i2.85

68. Becker, M. M., Graham, R. L., Donaldson, E. F., Rockx, B., Sims, A. C., Sheahan, T., Pickles, R. J., Corti, D., Johnston, R. E., Baric, R. S., and Denison, M. R. (2008) Synthetic recombinant bat SARS-like coronavirus is infectious in cultured cells and in mice. Proc Natl Acad Sci U S A 105, 19944-19949. doi: 10.1073/pnas.0808116105 